Review

\title{
Multi-flexible fiber flows: A direct-forcing immersed boundary lattice-Boltzmann lattice-spring approach
}

\author{
Yihsin Tanga ${ }^{a}$, Tai-Hsien $\mathrm{Wu}^{\mathrm{a}}$, Guo-Wei He ${ }^{\mathrm{b}}$, Dewei Qi ${ }^{\mathrm{a}, *}$

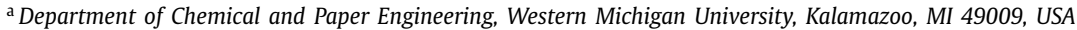 \\ ${ }^{\mathrm{b}}$ State Key Laboratory of Nonlinear Mechanics, Institute of Mechanics, Chinese Academy of Sciences, Beijing 100080, PR China
}

\section{A R T I C L E I N F O}

\section{Article history:}

Received 15 September 2016

Revised 26 October 2017

Accepted 3 November 2017

Available online 8 November 2017

\section{Keywords:}

Lattice Boltzmann equation

Lattice spring model

Flexible fibers

Fiber network

Drainage rate

Immersed boundary method

Rigidity

Flexibility

\begin{abstract}
A B S T R A C T
We demonstrate that a lattice-Boltzmann lattice-spring method can be used to simulate a dynamic behavior of a suspension of a large number of flexible fibers in finite Reynolds number flows. In the method, lattice-Boltzmann equation is adopted to simulate fluid velocity and vorticity while lattice-spring model with three-body forces can be employed to model the bending deformation of solid bodies. In order to realize the non-slip boundary condition, a forcing term is simply calculated by using the Newtonian second law and imposed with an immersed boundary scheme. The method is validated by comparing the present results with experiments and existing theories and methods. Subsequently, the method is applied to simulate a dynamic process of flexible fibers settling on a static or moving screen/wire net while a fiber mat is simultaneously built over the screen and resists fluid flowing. The number of fibers, fiber density and flexibility, and ratio of the relative velocity of the screen/wire to fluid can be systematically varied at different levels. Their influences on drainage rate are computed and evaluated.
\end{abstract}

(c) 2017 Elsevier Ltd. All rights reserved.

\section{Introduction}

There are extensive applications of particulate flows in chemical engineering (fluidized bed in reactor, particle separation, screening, filtration) and sciences (biological particle coagulation and dispersion, blood flows and cell adhesion, sands sedimentation in rivers), and in various industries (pharmaceutical, petroleum, food process, paper industry etc.). Due to the importance, engineers and scientists have paid a great attention to the subject of the particulate flows in both experimental and theoretical studies. Most investigations currently focus on the rigid particle-fluid problems. Very few studies are reported on deformable particle-flow problems. Some direct simulation methods are developed to model the solid particle motion in fluids. Among the traditional finite element method, the most popular one is an arbitrary Lagrangian Eulerian scheme (Hu, 1996) based on Galerkin finite element approach. In this method, the meshes are moving with solid particles and the meshes need to be re-meshed when unacceptable element deformation is detected. It is obvious that the re-meshing process largely increases computational load and limits to a system with a small number of particles. This method is recently ex-

\footnotetext{
* Corresponding author.

E-mail address: dewei.qi@wmich.edu (D. Qi).
}

tended to treat deformable particles (Gao et al., 2011, 2013), only two dimensional cases at a very low Reynolds number were reported. Later, Glowinski et al. (1999) with others (Glowinski et al., 2001; Patankar et al., 2000; Wan. and Turek., 2006) presented a Lagrange-multiplier-based fictitious-domain method for the motion of large numbers of rigid particles in a Newtonian fluid. Their finite element formulation allows use of a fixed structured grid and avoids re-mesh process. However, their reports are limited to rigid solid particles.

Peskin (1977) was the first to propose an immersed boundary method (IBM) to treat deformable bodies associated with blood flow. Later, the IBM had been applied to particulate flows (Fogelson and Peskin, 1988). In Peskin's method, regular Eulerian grids are operated for fluid domain and the Lagrangian grids of deformable particles move over the fixed fluid grids. A forcing term is added on the interface between the fluid and solid to force the solid boundary velocity equal to the fluid velocity (non-slip boundary condition) through Dirac delta function. For fluid field, the Navier-Stokes equations can be solved either by traditional computational fluid dynamics (CFD) or by a latticeBoltzmann method (LBM) (Succi, 2001). The solid particles can be either rigid or deformable.

Ladd (1994) had firstly proposed a moving boundary condition in the LBM and applied it to particulate flows. Later, Koch and 
others (Aidun and Clausen, 2010; Aidun et al., 1998; Benzi et al., 1992; Duenweg and Ladd, 2009; Feng and Michaelides, 2004, 2005; Koch and Ladd, 1997; Luo et al., 2017; Qi, 1999; Qi and Gordnier, 2015; Qi et al., 2014a, 2014b, 2010; Whittington, 1998) had demonstrated that the LBM is the most simple and efficient method to simulate particle suspensions. Unlike traditional CFD, one of features of the lattice-Boltzmann method (LBM) is that micro-kinetic nature of fluid particle collision and streaming, similar to convection-diffusion of fluid momentum, is presented through the lattice-Boltzmann equation at every time step. The method allows ones to simulate flows efficiently in complex geometrical solid boundaries. The main features and advantages as well as disadvantages of LBM are briefly summarized in a recent article by Succi (2015). Feng and Michaelides (2004) were the first to combine the advantage of LBM with IBM to deal with rigid particles. For deformable particles, a lattice-spring model was proposed to handle the deformation of a flexible body by Buxton et al. (2005). In their method, the deformable solid body is discretized as individual particles located in a regular lattice and connected by a two-body spring force between two neighboring particles as a bond. The two-body force can deal with extension and compression but cannot accurately handle the bending deformation, because the two-body central force is a function of distance between two neighboring solid particles and does not provide any bonding angle information between two adjacent bonds or springs. Wu and Aidun (2010a, 2010b, 2010c) took Buxton's spring model in their LB simulation. Subsequently, a threebody force with angular information between two adjunct bonds was added into the lattice-spring model to handle bending deformation by Wu et al. (2014). Other authors such as Duenweg and Ladd (2009) and Basagaoglu et al. (2013, 2008) also utilized LBM and bead-spring model to simulate the soft materials in flows.

Unfortunately, both Wu and Aidun (2010a, 2010b, 2010c) and $\mathrm{Wu}$ et al. (2014) used the same forcing term as those by He et al. (1998) and Feng and Michaelides (2004) to take account of the interactions between fluids and solid particles. Guo et al. (2002) proved that this type forcing (body force) term cannot be operated for an unsteady body force case because the Navier-Stokes equations cannot be entirely recovered due to extra-terms caused by the spatial and temporal variation of the body force and they proposed a new forcing term, which enables the LB equation to recover the Navier-Stokes equations with a second-order accuracy for unsteady, non-uniform body forces. This new force term is called split-force and used by Kang and Hassan (2011) and Delouei et al. (2014) for rigid particle cases. We will adopt the direct split-forcing term by Guo et al. (2002) in this work.

Among all particulate flows, a slender fiber system is difficult to cope with due to a large aspect ratio and deformability, which may complicates the fluid-solid interaction, although a numerical method based on a slender body theory has been extensively applied to simulate multi fibers by Butler and others (Butler and Shaqfeh, 2002; Saintillan et al., 2006; Shin et al., 2006, 2009). Their reports were limited either to very low Reynolds number or to rigid fibers. To consider inertial effect, some numerical methods for finite Reynolds flows were also developed (Dahlkild, 2011; Kuusela et al., 2001; Zhang et al., 2013) for rigid fibers. Lindstrom and Uesaka reported a flexible fiber model in fluid (Lindström and Uesaka, 2007, 2008). They used vorticity-vector potential form of the Navier-Stokes equations for fluid while fibers were modeled as a chain of segments interacting with themselves through a contact force. They also considered short and long-range hydrodynamic interactions. Their semi-dilute regime results for rheological properties showed deviations from hydrodynamic interaction theories. Salahuddin et al. $(2012,2013)$ used a lattice-Boltzmann equation to simulate fluid and fibers were modeled as a chain of rods where 4 nodes were distributed on the circumference of each cross section of the fiber. The interaction between fluid and fiber was treated by an external boundary force. However, the direct relationship between the fiber rigidity and structure of the fiber and the effects of rigidity on motion were not reported.

In order to effectively investigate effects of fiber rigidity on motion, we present a direct-forcing immersed boundary latticeBoltzmann lattice-spring approach to simulate a suspension with a large number of flexible fibers. The approach is comprised of the following portions, and its advantage is briefly summarized as follows:

1. The LBM will be employed to simulate fluid flow behavior and structure on fixed Eulerian nodes. Most importantly, the Boltzmann equilibrium distribution function physically represents a natural distribution of fluid particles and is presented at every time step so that the error is controlled. In contrary, the traditional CFD is a sole numeric approximation and lacks such the equilibrium function. The algorithm of the LB method for fluid domain is extremely simple and consists of only two operations, collision and streaming. This simplicity essentially comes from two major reasons. First, when one derives the Navier-Stokes equation from the LB equation, the fluid distribution function is expanded to the second order of the small Knudsen number (the ratio of the mean free length to the characteristic spatial scale of the whole fluid system), called the Chapman-Enskog expansion or multi-scale analysis, so that collision, convection, and diffusion time scales are separated. This greatly simplifies the algorithm. Second, the fluid collision matrix of the LB equation is also linearized by either the Bhatnagar-Groos-Krook (BGK) approximation or multi relaxation time models.

2. The distinct feature of the LB method is well known that the computation efficiency in the LB method is much higher than that in the traditional finite element and differential methods, due to the parallel nature of the LB algorithm itself, where only local information at one grid needs to be transferred to its neighboring grids, and the same simple operations are repeated on all grids. The code is easy to be parallelized in a GPU based CUDA code (Wu and Qi, 2017; Wu et al., 2017). Also, the fluid grids are fixed so that the method is easy to handle moving and flexible solid boundaries.

3. The lattice-spring model with three-body forces is utilized to mimic motion of discretized flexible fibers where moving Lagrangian solid particles are connected by elastic harmonic springs and angular bonds. This fiber model can consider not only extension, compression, but also bending deformation. It is noted that two body central force cannot treat bending deformation. The deformation and motion of a flexible fiber are treated with an elastic model without resorting to a nonlinear Euler Bernoulli beam equation, which is a 4 th order differential equation. The present approach allows us to accurately simulate a large number of fibers in a finite Reynolds number flow. In addition, the lattice-spring model can be used to construct solid particles in any shape, such as rectangular, plate-like, nonspherical, spherical, cylindrical, etc., to treat deformation easier due to the use of elastic springs, and to accurately handle fibers with a large aspect ratio.

4. The split-force term proposed by Guo et al. (2002) will be adopted to correctly address interactions between solids and flows. This algorithm takes into account the nonlinear effects of lattice discrete and unsteady interaction forces on the solidfluid interfaces, and the Navier-Stokes equations are exactly recovered.

5. The fluid force or the force on solid nodes for non-slip boundary condition is simply calculated from the difference be- 
tween unforced velocity (defined in Section 2.3) and the solid boundary velocity to avoid solving high dimensional equations (Wu and Shu, 2010), which is not realistic for a threedimensional case.

6. Immersed Boundary method with the Dirac delta function is used to obtain the fluid velocity at the solid boundary node and distributes the fluid force into fluid nodes.

The validation for the direct-forcing immersed boundary latticeBoltzmann lattice-spring approach is carried out by comparing our simulation results with 1) a flexible particle method; 2) Jeffery theory; 3) Forgacs and Mason's experiments; and 4) the theory of Wiggins, Riveline and Goldstein.

Subsequently, we apply this approach to simulate fibers settling on a moving screen/wire net and to evaluate the drainage rate as a function of time. It is well known that wire screens or filters are popularly employed to separate solid materials from fluids and to classify solid particles into different categories in terms of size or density. While the fluids flow through the screen openings or holes, the solid particles may remain on the top surface of the filter/screen and form a solid particle cake or mat. This technique has been employed by various industries. For example, the paper industry uses screen to collect and form a fiber sheet in the forming section of a paper machine. A washing machine also uses filters to collect cleaned solid particles and reject dirty water. Efficiencies of formation of fiber mat and of washing machine are directly related to the fluid drainage rate. The water drainage is driven not only by the hydrodynamic forces but also by the gravity of both fluid and solid particles and resisted by the solid particle surfaces. When a fiber mat becomes thicker during settling, the resisting force increases and the drainage rate is getting smaller. The drainage rate will be affected by concentrations of fibers, specific surface areas, fiber flexibility, screen structures, opening ratios, the ratios of screen-to-fluid velocity, temperatures, $\mathrm{pH}$ values (which may change the electrical charges of the solid particles) etc. The present work will focus on studies of effects of fiber concentrations, fiber flexibility, and screen-to-fluid speed ratios on the drainage rates of a screen by using the present approach. Although there are hundreds of articles on topics of fiber suspensions (Koch, 1994; Koch and Shaqfeh, 1989, 1991; Qi, 2006; Qi et al., 2014b; Shin et al., 2009), very few of studies on drainage rates exist in the literature. We will demonstrate that the forming process of a flexible fiber network over a wire net/screen can be effectively simulated and that the drainage rate is a nonlinear function of time and is significantly affected by fiber's mass.

In the simulations, up to 500 of fibers are used. The fiber concentrations, fiber densities and flexibility, and screen-to-fluid speed ratios, are systematically varied at different levels and the drainage rate and fluid structure are computed during the fibers settle and the fiber networks are built over the screen.

The present approach is introduced in Section 2. The validation is presented in Section 3. Section 4 will introduce simulation setup and present the results of the effects of fiber concentrations, flexibility, flow-to-wire shear ratios, and screen opening ratios on drainage rates. The Section 5 will make conclusions.

\section{Simulation method}

\subsection{Lattice-Boltzmann method}

A group of lattice nodes is used to represent fluid. Each node has distribution functions $f_{\sigma}$ and discrete micro-velocity $\mathbf{e}_{\sigma}$, where $\sigma$ depends on the chosen lattice model. The Boltzmann equation with BGK (Bhatnagar et al., 1954) single relaxation time model is

$$
\begin{aligned}
& f_{\sigma}\left(\mathbf{r}^{l}+\mathbf{e}_{\sigma}, t+\delta t\right) \\
& \quad=f_{\sigma}\left(\mathbf{r}^{l}, t\right)-\frac{1}{\tau}\left[f_{\sigma}\left(\mathbf{r}^{l}, t\right)-f_{\sigma}^{e q}\left(\mathbf{r}^{l}, t\right)\right]+\delta t \mathbf{F}_{\sigma}\left(\mathbf{r}^{l}, t\right)
\end{aligned}
$$

where $\tau$ is the relaxation time; $\mathbf{F}_{\sigma}\left(\mathbf{r}^{l}, t\right)$ is a body force; $f_{\sigma}^{e q}\left(\mathbf{r}^{l}, t\right)$ is the equilibrium distribution function at node position $\mathbf{r}^{l}$ and time $t$ as

$f_{\sigma}^{e q}=\omega_{\sigma} \rho_{f}\left\{1+3\left(\mathbf{e}_{\sigma} \cdot \mathbf{u}\right)+\frac{9}{2}\left(\mathbf{e}_{\sigma} \cdot \mathbf{u}\right)^{2}-\frac{3}{2}(\mathbf{u} \cdot \mathbf{u})\right\}$,

where $\mathbf{u}$ is the fluid velocity. In this simulation, the D3Q15 lattice model is applied and the discrete velocities are given by

$\mathbf{e}_{\sigma}= \begin{cases}(0,0,0), & \sigma=0 \\ ( \pm 1,0,0),(0, \pm 1,0),(0,0, \pm 1), & \sigma=1 \\ ( \pm 1, \pm 1, \pm 1), & \sigma=2\end{cases}$

and the weight coefficients are

$\omega_{\sigma}= \begin{cases}\frac{2}{9}, & \sigma=0 \\ \frac{1}{9}, & \sigma=1 \\ \frac{1}{72}, & \sigma=2\end{cases}$

According to Guo et al. (2002),

$\mathbf{F}_{\sigma}=\left(1-\frac{1}{2 \tau}\right) \omega_{\sigma}\left(\frac{\mathbf{e}_{\sigma}-\mathbf{u}}{c_{s}^{2}}+\frac{\mathbf{e}_{\sigma} \cdot \mathbf{u}}{c_{s}^{4}} \mathbf{e}_{\sigma}\right) \cdot \mathbf{F} ;$

where $\mathbf{F}$ is the body force and its component in the $\sigma$ direction is $\mathbf{F}_{\sigma} ; c_{s}^{2}=1 / 3$ where $c_{s}$ is the sound speed; the fluid density $\rho_{f}$ and momentum density $\rho_{f} \mathbf{u}$ are given by

$\rho_{f}=\sum_{\sigma} f_{\sigma}$

$\rho_{f} \mathbf{u}=\sum_{\sigma} f_{\sigma} \mathbf{e}_{\sigma}+\frac{1}{2} \delta t \mathbf{F}$

The kinematic viscosity $v$ is related to the relaxation time $\tau$ and is given by

$v=\frac{1}{3}\left(\tau-\frac{1}{2}\right) \delta t$

\subsection{Lattice-spring model}

A flexible fiber is modeled by the lattice-spring method. In the method, the fiber is discretized as individual particles, which are connected by bonding harmonic elastic springs and angular elastic springs to ensure that three body force is added into model so that it can handle the bending deformation (Wu et al., 2014). Thus, the spring energy $U^{s}$ acted on the $i$ th particle is given by

$U_{i}^{s}=\frac{1}{2} k_{s} \sum_{j}\left(\mathbf{r}_{i j}-\mathbf{r}_{0 i j}\right)^{2}$

where $k_{s}$ is the spring coefficient; $\mathbf{r}_{0 i j}$ is the equilibrium length of the spring between two neighboring particles $\mathrm{i}$ and $\mathrm{j} ; j$ are the neighboring solid particle of the $i$ th solid particle; $\mathbf{r}_{i j}=\mathbf{r}_{i}-\mathbf{r}_{j}$. The angular energy $U^{a}$ is given by

$U_{i}^{a}=\frac{1}{2} k_{a} \sum_{j} \sum_{k, k \neq j}\left(\theta_{i j k}-\theta_{0 i j k}\right)^{2}$

where $k_{a}$ is the angular bonding coefficient; $j, k$ are the nearest neighboring solid particles of $i$ th solid particle; $\theta_{i j k}$ is the angle between the bonding vectors $\mathbf{r}_{i j}$ and the bonding vector $\mathbf{r}_{i k} ; \theta_{0 i j k}$ is the corresponding equilibrium angle. The elastic force $\mathbf{F}_{i}^{e}$ on the ith solid particle can be computed from the gradient of the total energy.

$\mathbf{F}_{i}^{e}=-\nabla\left(U_{i}^{s}+U_{i}^{a}\right)$

The elastic modulus of the solid body can be related to the spring and angular coefficients. The relationship between elastic modulus 
$E$ and shear modulus depends on lattice structure. For a cubic lattice structure without diagonal bonds, we have

$E=\frac{k_{s}}{r_{0}}$

where $r_{0}$ is the distance between particles $\mathrm{i}$ and $\mathrm{j}$ (Wu et al., 2014) if the solid material is isotropic.

For a single bonded fiber model, it can be considered as a flexible beam. Thus, the rigidity EI can be related to the bonding angle $\theta_{i j k}$ through Euler-Bernoulli equation,

$\mathbf{M}=-E I \frac{1}{R}=-E I \frac{\left(\theta_{i j k}-\theta_{0 i j k}\right)}{r_{0}}$

where $\mathbf{M}$ is the torque in the direction of $\frac{\mathbf{r}_{i j}}{r_{i j}} \times \frac{\mathbf{r}_{i k}}{r_{i k}} ; r_{i j}=\left|\mathbf{r}_{i}-\mathbf{r}_{j}\right| ; R$ is a radius of the arch composed of $\mathbf{r}_{i j}$ and $\mathbf{r}_{i k}$. The minus sign denotes the torque direction against the deformation angle increase. From Eq. (13), we have

$E I=k_{a} r_{0}^{2}$.

The rigidity can be normalized by

$E I^{*}=\frac{E I}{\rho_{f} V_{0}^{2} H L}$

where $V_{0}$ is a reference velocity, the driving lip velocity for a shear flow or the maximum velocity for sedimenting flow; $H$ is the volume of the solid fiber.

\subsection{Interaction between solid and fluid}

In order to force the fluid velocity equal to the solid boundary velocity at the fluid-solid interface, a direct forcing-force $\mathbf{F}$ should be added to the unforced fluid velocity $\mathbf{u}^{*}\left(\mathbf{r}^{b}, t\right)$ at the solid boundary position $\mathbf{r}^{b}$ and make their momentum summation equal to the solid boundary velocity $\mathbf{U}^{b}\left(\mathbf{r}^{b}, t\right)$ according to the Newtonian second law. This is

$\rho_{f} \mathbf{u}\left(\mathbf{r}^{b}, t\right)=\rho_{f} \mathbf{u}^{*}\left(\mathbf{r}^{b}, t\right)+\delta t \mathbf{F}=\rho_{f} \mathbf{U}^{b}\left(\mathbf{r}^{b}, t\right)$

where $\mathbf{u}\left(\mathbf{r}^{b}, t\right)$ is the forced fluid velocity at $\mathbf{r}^{b}$. The unforced velocity is calculated by

$\rho_{f} \mathbf{u}^{*}=\sum_{\sigma} f_{\sigma} \mathbf{e}_{\sigma}$

Therefore, the direct forcing force on fluid can be calculated by

$\mathbf{F}\left(\mathbf{r}^{b}, t\right)=\frac{\rho_{f}\left(\mathbf{U}^{b}\left(\mathbf{r}^{b}, t\right)-\mathbf{u}^{*}\left(\mathbf{r}^{b}, t\right)\right)}{\delta t}$.

The force acting on the solid boundary node by fluid is $-\mathbf{F}\left(\mathbf{r}^{b}, t\right)$. The unforced fluid velocity $\mathbf{u}^{*}$ at the position of the solid boundary node $\mathbf{r}^{b}$ is presented by

$\mathbf{u}^{*}\left(\mathbf{r}^{b}, t\right)=\int_{\Pi} \mathbf{u}^{*}\left(\mathbf{r}^{l}, t\right) D\left(\mathbf{r}^{l}-\mathbf{r}^{b}\right) d \mathbf{r}^{l}$

where $\mathbf{r}^{l}$ is a variable.

Since the fluid node position may not be coincided with the solid particle position, the fluid velocity on the boundary solid node can be approximately extrapolated from the fluid velocity of the surrounding solid boundary node by using a discrete Dirac Delta function (Peskin, 2002)

$D(\mathbf{r})= \begin{cases}\frac{1}{64 h^{3}}\left(1+\cos \frac{\pi x}{2 h}\right)\left(1+\cos \frac{\pi y}{2 h}\right)\left(1+\cos \frac{\pi z}{2 h}\right) & |\mathbf{r}| \leq 2 h \\ 0 & \text { otherwise }\end{cases}$

where the $h$ is the one lattice unit length. The fluid nodes are within a spherical volume $\Pi$ of a radius of $2 h$, centered at a given solid node $\mathbf{r}^{b}$.
It is noted that the fluid forcing force needs to be calculated by using Eq. (18). Its physical meaning is that based on momentum conservation, the forcing force is added to unforced fluid particle and ensure its velocity equal to the solid particle velocity. Only after the forcing force is obtained from Eq. (18), fluid velocity can be calculated by using Eq. (7), which cannot be used to define the forcing force as (Delouei et al., 2014; Kang and Hassan, 2011) did incorrectly. The factor $1 / 2$ in the second term of the right side of Eq. (7) is used to eliminate the extra terms which do not exist in the Navier-Stokes equations. The correct procedure should follow three steps. The first step is to define the forcing force using Eq. (18). The second step is to calculate the fluid velocity by using the obtained forcing force and Eq. (7). The third step is to add the forcing term components $\delta t \mathbf{F}_{\sigma}$ into fluid following Eq. (1). The three steps complete a forcing-force addition procedure. For the third step, the discrete Dirac delta function is utilized again to distribute the reaction force to the surrounding fluid nodes

$\mathbf{F}\left(\mathbf{r}^{l}, t\right)=\int_{\Gamma} \mathbf{F}\left(\mathbf{r}^{b}, t\right) D\left(\mathbf{r}^{l}-\mathbf{r}^{b}\right) d \mathbf{r}^{b}$

where $\mathbf{F}\left(\mathbf{r}^{l}, t\right)$ is the distributed force and $\Gamma$ is a spherical volume of a radius of $2 h$, located at $\mathbf{r}^{l}$.

In addition, to avoid two fiber overlap during simulations, the repulsive portion of the Lennard-Jones potential is employed between the solid particles in different fibers.

$U_{L J}=\epsilon\left(\frac{\Sigma}{\mathbf{r}_{i j}}\right)^{12}$

$\Sigma$ is a force interaction diameter and $\epsilon$ is the parameter of the intensity of the repulsion. The total force on a discretized solid particle $i$ is

$\mathbf{F}_{i}^{T}=-\mathbf{F}_{i}\left(\mathbf{r}^{b}\right)+\mathbf{F}_{i}^{e}+\mathbf{F}_{i L J}$.

where $\mathbf{F}_{i L J}$ is the force on the ith particle due to the repulsive Lennard-Jones potential.

It is critical to select correct values of the parameters $\Sigma$ and $\epsilon$ to assure the approximation of momentum conservation during collision. A simulation is conducted for a head-to-head collision between two spheres of the diameter $D=5$. The first sphere with a velocity of $V_{0}=0.005$ collides with the second sphere at rest. In the simulation, $\Sigma=2$ and $\epsilon=8.06 \times 10^{-6}$ are optimally chosen and the potential cut off is the same as $\Sigma=2$. After the collision, the first sphere becomes stationary and the second sphere obtains an equal velocity, as shown in Fig. 1, indicating that the selected values of the two parameters ensure the momentum exchange between the two spheres. The same values of $\Sigma$ and the $\epsilon$ are taken for the simulations of drainage rates in this work. The simulations are stable and no any particle overlapped case is detected.

Next, the leap frog algorithm is taken to update the position and velocity of each solid particle at each time step by using Newtonian mechanism. The details about the leap frog algorithm is referred to Chapter 3 of the book by Allen and Tildesley (1987).

In summary, the procedure for implementation of simulation follows

1. compute the unforced velocity $\mathbf{u}^{*}\left(\mathbf{r}^{l}, t\right)$ by using Eq. (17), calculate the unforced velocity $\mathbf{u}^{*}\left(\mathbf{r}^{b}, t\right)$ using Eq. (19), then calculate the direct forcing force by Eq. (18);

2. compute velocity by using Eq. (7), distribute the forcing force to fluid using Dirac delta function again, then calculate the force components by using Eq. (5);

3. follow Eq. (1) for collision, add forcing component and streaming;

4. use the total force of Eq. (23) to update the solid particle position and velocity by using a half leap frog algorithm;

5. iterate; 


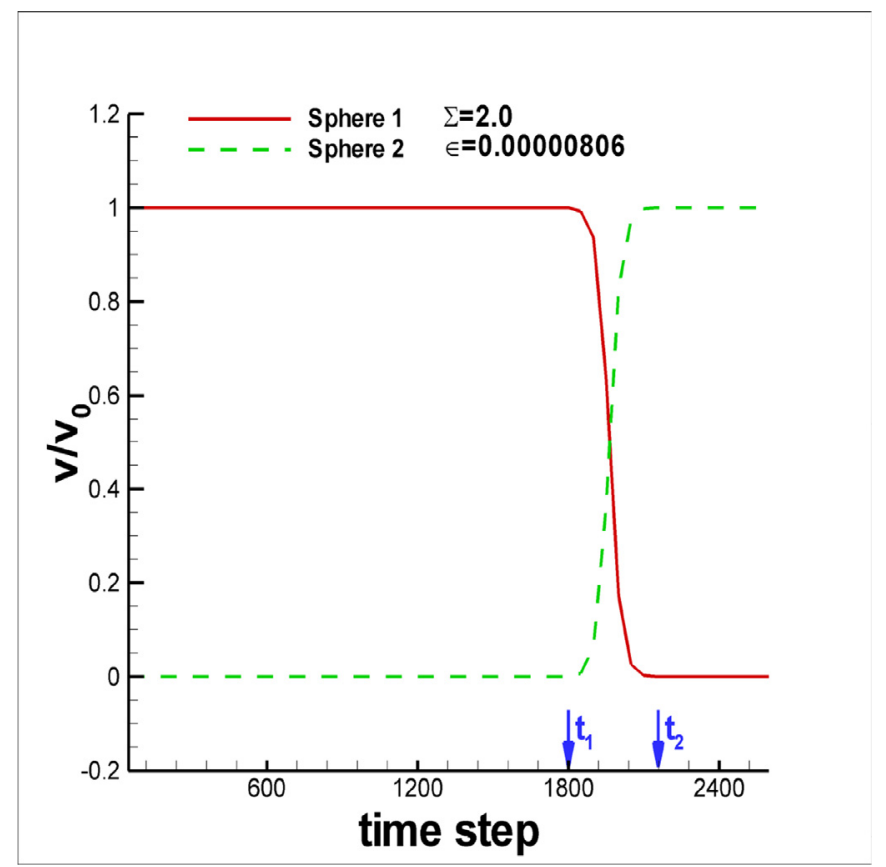

Fig. 1. The velocity exchange during the collision between two spheres of $D=5$ as a function of time is shown. The simulation box size is $32 \times 45 \times 32$ and $\Sigma=2$ and $\epsilon=8.06 \times 10^{-6}$ are selected. The $\mathrm{LJ}$ repulsive force is turned on between time $t_{1}$ and $t_{2}$. The velocity $V$ is normalized by the initial velocity $V_{0}$.

\section{Validation}

\subsection{Comparison with Forgacs and Mason's experiments}

For motion of a flexible fiber in a laminar shear fluid, Forgacs and Mason (1959a,b) conducted a series of experiments, they observed a "springy" orbit due to a shear-induced buckling force when the rigidity was smaller or flexibility was larger than a critical value. Forgacs and Mason found that as the flexibility increased, a "snake" rotation appeared. That is, one end of fiber bent and other end straightened during rotation. When the fiber was highly symmetrical, the ends of the fiber bent simultaneously in opposite directs so that the fiber formed a " $\mathrm{S}$ " shape, and then straightened along the fluid streaming direction. Both " $\mathrm{C}$ " and " $\mathrm{S}$ " shapes were found and confirmed in other authors' simulation results (Joung et al., 2001; Lindström and Uesaka, 2008; Stockie and Green, 1998). As the flexibility continuously increased, a coiled orbit without entanglement occurred. When flexibility reached a high level, a coiled orbit with entanglement arose. In their experiments, the flexibility might be increased by increasing either fiber length or shear rate.

According to Burgers' theory (Burgers, 1938), the compressive force $F_{H}$, induced by the shear flow, drives fiber buckling while the rigidity EI resists the deformation. Forgacs and Mason (1959a) pointed out that the maximum compressive force on the rod cross section is $F_{H}^{m} \propto \frac{\lambda \nu \rho_{f} L_{0}^{2}}{\ln \left(2 r_{e}\right)-1.75}$ where $L_{0}=0.5 L$. Therefore, we can define a non-dimensional ratio of buckling force-to-rigidity as

$\kappa=\frac{F_{H}^{m} L_{o}}{E I / L_{0}}=\frac{\lambda v \rho_{f} L_{0}^{3} /\left(\ln \left(2 r_{e}\right)-1.75\right)}{E I / L_{0}}$

where the numerator is proportional to the maximum of the buckling force, on the cross section at the middle of the axis, which drives fiber buckling while the dominator is the rigidity which resists the deformation. Thus, as the force-to-rigidity number $\kappa$ increases, the flexibility and deformation increase.

We conduct several simulations at different force-to-rigidity numbers with an increase in flexibility by increasing shear rate, fiber length and reducing fiber rigidity. The purpose is to recover a variety of rotation orbits discovered by Forgacs and Mason who used a filament with a very large aspect ratio as high as $r_{e}=833$. Due to computation power limitation, we focus on the comparison with their found phenomena by using much smaller aspect ratio fibers in our simulations.

An undisturbed shear fluid velocity field is imposed by $u=z \lambda \mathbf{e}_{x}$ in simulations and the coordinate origin is at the center of the simulation box where $\mathbf{e}_{\mathbf{x}}$ is the unit vector of the X-axis. The simulation results of fiber rotation under the shear flow are shown in Fig. 2 for a springy orbit where the fiber size of $(1,1,50)$, simulation box size of $(255,63,127)$, and $\kappa=25.05$ are used.

At a large flexibility, the simulation results are shown in Fig. 3 for snake orbits. The top row is for a " $\mathrm{C}$ " shape and the bottom row is for a " $\mathrm{S}$ " shape orbit where $\kappa=322.54$ is used. The " $\mathrm{C}$ " shape is obtained when the mass center of a fiber is initially placed above the center line of the simulation box and the fiber has an angle with the center line while the " $S$ " shape is obtained when the initial fiber position is symmetrically located at the center. When the flexibility further increases, a coiled orbit appears. The results of the coiled orbits are shown in Fig. 4 without entanglement where $\kappa=8321.57$ is used. For this case, a helix rotation is observed in the projection on the XY-plane as shown in Fig. 5 from our 3D simulations, which is shown in Fig. 6. The fiber helically rotates about the shear vorticity axis in the Y-direction without straightening. As the flexibility continuously increases to a very large level, a coiled orbit with entanglement is obtained in the simulation results as shown in Fig. 7 where fiber size of $(1,1,200)$, simulation box size of $(511,63,127)$, and $\kappa=445,545.11$ are utilized. A threedimensional view is shown in Fig. 8. All experimental orbits found by Forgacs and Mason are recovered in the present simulations.

\subsection{Comparison with the theory of Wiggins Riveline and Goldstein}

To further quantitatively valid the present method, the flapping motion of a flexible filament is simulated in fluids by using the present method. The simulation results of the wave pattern of the filament are compared with an existing theoretical results by Wiggins et al. (1998). Two elastic filaments with sizes of $2 \times 2 \times 20$ and $2 \times 2 \times 40$ are constructed and used, respectively. Their aspect

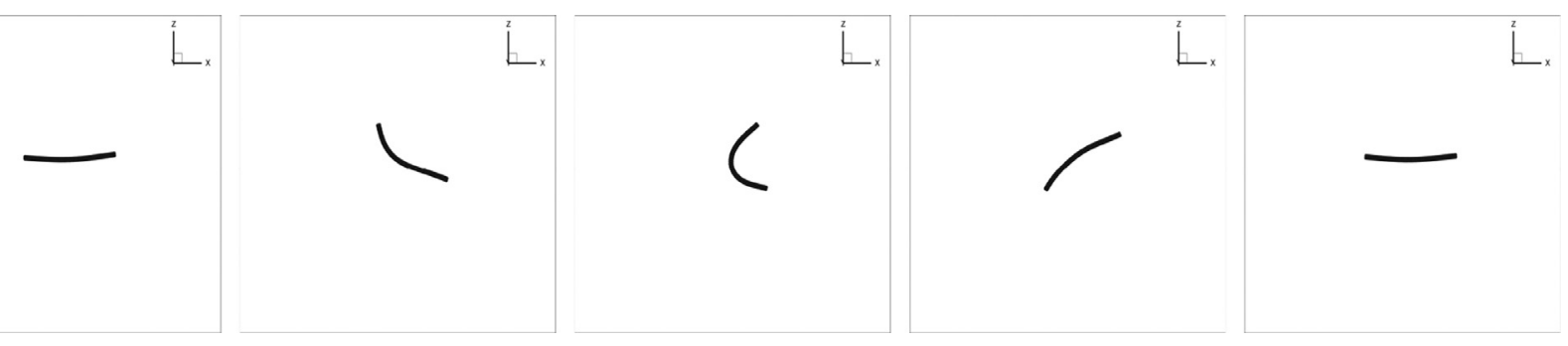

Fig. 2. A springy orbit at different time instants at $\kappa=25.05$. 


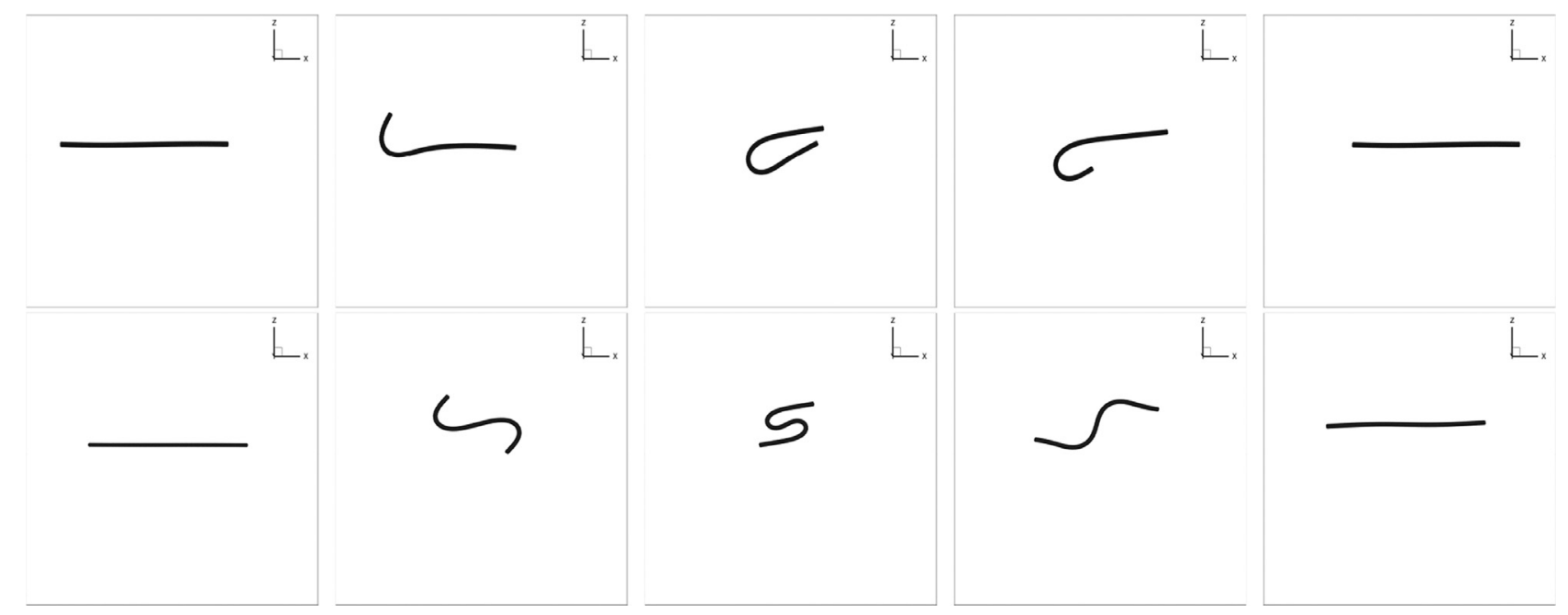

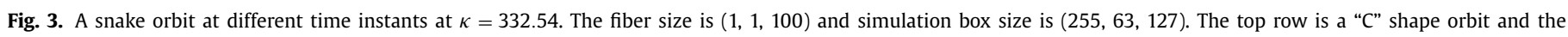
bottom row is a "S" shape orbit.

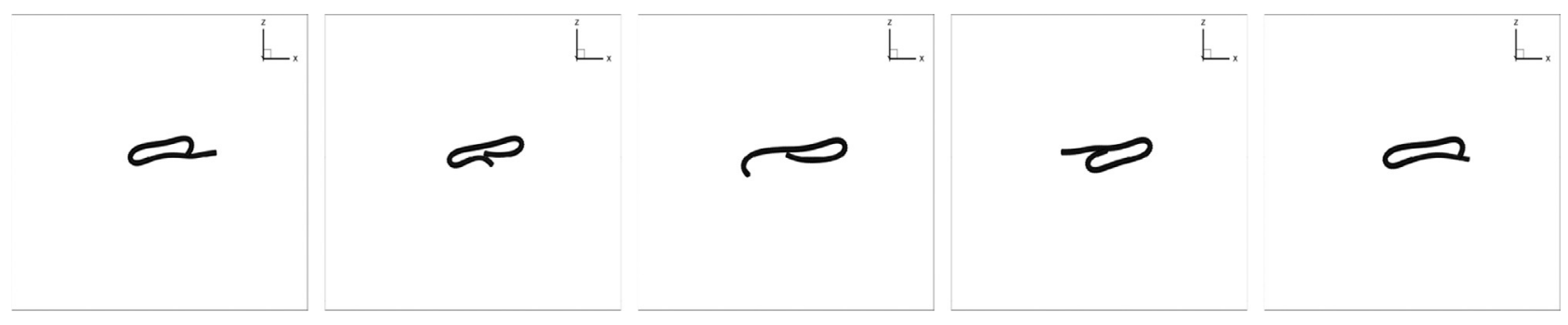

Fig. 4. A coiled rotation without entanglement at $\kappa=8321.57$. The fiber size is $(1,1,100)$ and simulation box size is (255, 63, 127).

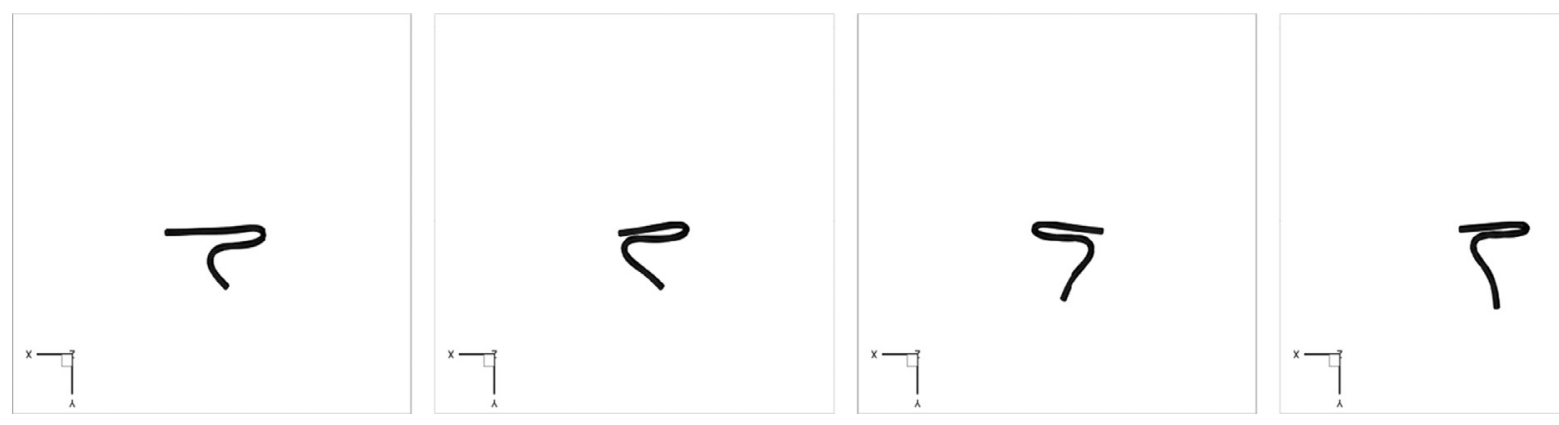

Fig. 5. Helix orbit projected on the XY-plane at different time instants at $\kappa=8321.57$ for the case of coiled rotation without entanglement.

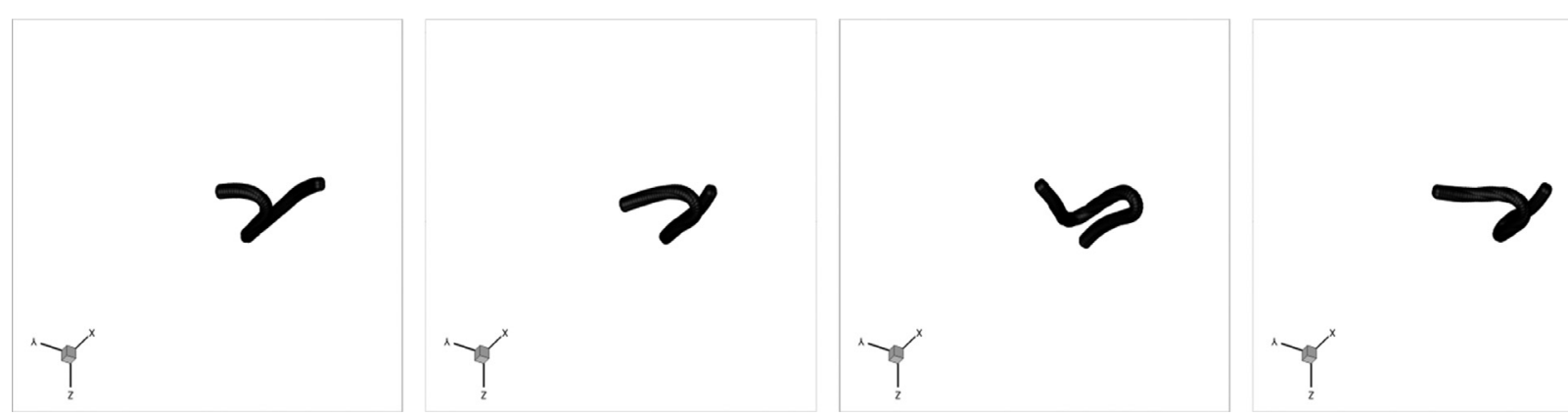

Fig. 6. Three dimensional view for helix orbits at different time instants at $\kappa=8321.57$ for the case of coiled rotation without entanglement.

ratios of $L / D$ are 10 and 20 , where $L$ is the filament length; $D$ is the diameter. The cross section is a square of $2 \times 2$. The simulation box size is $\left(N_{x}, N_{y}, N_{z}\right)=(13 D, 8.05 L, 12 L)$. A periodic condition is imposed in the X-, Y-, Z-directions. The density of the filament is the same as the fluid density. The filament is located in the center of the simulation box. A driven point is located at the left end of the filament. The motion of the driven point follows a pre-described harmonic function with a transverse amplitude

$Z=Z_{0} \sin \omega t$

where $Z_{0}$ is the amplitude; angular velocity $\omega=2 \pi f$. The filament right end is allowed to move freely.

Based on the elastohydrodynamics, when the inertia can be ignored, i.e., the Reynolds number is very small, 


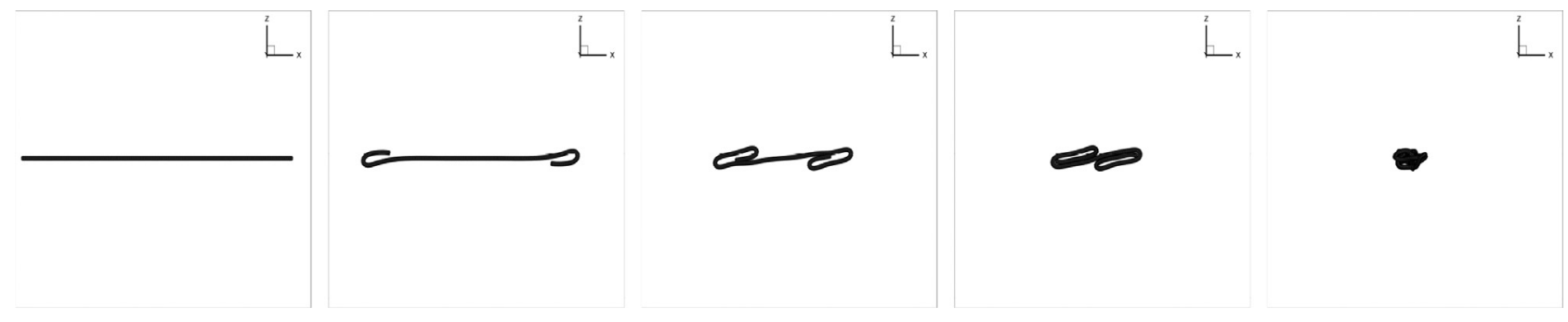

Fig. 7. Coiled orbits with entanglement at different time instants at $\kappa=445545.11$.

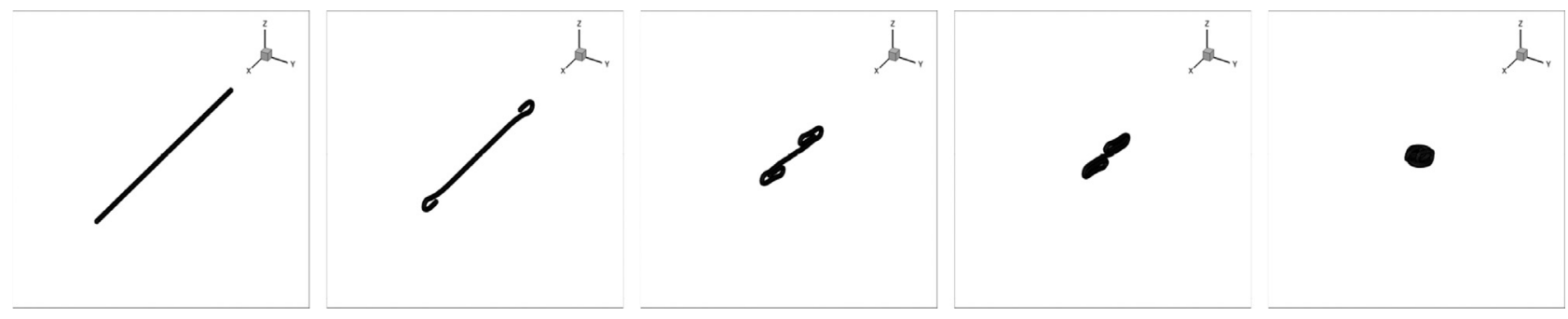

Fig. 8. Three-dimensional views of the coiled orbits with entanglement at different time instants at $\kappa=445,545.11$.

Wiggins et al. (1998) obtained a solution of wave pattern, which is given by

$Z=\frac{1}{2} Z_{0}\left[e^{-\tilde{C} \eta} \cos (\tilde{S} \eta+\omega t)+e^{-\tilde{S} \eta} \cos (\tilde{C} \eta-\omega t)\right]$

where $y$ is the horizontal distance from the driving point; $\tilde{C}=$ $\cos \left(\frac{\pi}{8}\right) ; \tilde{S}=\sin \left(\frac{\pi}{8}\right) ; \eta=y / l_{\omega}$. A characteristic length $l_{\omega}$ is defined by

$l_{\omega} \equiv\left(\frac{E I}{\omega \zeta_{\perp}}\right)^{\frac{1}{4}}$

where the drag coefficient $\zeta_{\perp}=\frac{4 \pi \mu}{\ln \left(\frac{L}{D}\right)+\frac{1}{2}}$ is derived from the slender body theory, $\mu$ is the viscosity while the sperm number is defined by

$S p \equiv \frac{L}{l_{\omega}}$

The simulation results of the wave patterns of the filament at different time instants are compared with the theoretical results of Wiggins et al. (1998) in Fig. 9 for the cases of the fiber sizes of $2 \times 2 \times 20$ and $2 \times 2 \times 40$ where the axes are normalized by the length of the filament. The lines labeled as "theory" are plotted from the analytic solution of Eq. (26). The simulation results well agree with the theory, illustrating that the present developed method is reliable.

\section{Simulations and results}

\subsection{Forming of a fiber mat}

In a forming section of a paper machine, a sedimentation process and a screen or wire net are used to separate flexible solid fibers from water due to their sizes larger than screen opening. When the fibers settle down vertically by the gravity and are randomly distributed on the surface of the screen, a fiber mat is being build. In the initial stage of the sedimentation, fibers accelerates by the gravity and their velocity increases until the velocity arrives at a maximum. After passing the maximum, some of the fibers may land on the screen, a fiber mat is gradually built on the surface of the screen. The fiber velocity and flow rate or drainage rate decrease as the thickness of the fiber network increases due to the large flow resistance caused by the fiber mat itself.
The sedimentation behavior of the fibers is mainly determined by hydrodynamic force, and fiber elasticity and gravity. The fiber motion and its deformation can be approximated by a beam motion equation and fluid follows Navier-Stokes equations. The two equations can be non-dimensionalized if the length, velocity, time and force are re-scaled by a fiber length $L$, reference velocity $V_{0}$, time $L / V_{0}$, and $\mu V_{0} L$, respectively, where $\mu$ is the viscosity. The non-dimensional Navier-Stokes equations for fluids can be written by

$\nabla \cdot \mathbf{u}=0$

$R_{s d}\left(\frac{\partial \mathbf{u}}{\partial t}+\mathbf{u} \cdot \nabla \mathbf{u}\right)=-\nabla p+\nabla^{2} \mathbf{u}$

where $R_{s d}=V_{0} L / v$ is the particle Reynolds number, $p$ is the pressure, and the non-dimensional beam equation can be written by

$E I^{*} \frac{\partial^{4} w}{\partial l^{4}}+\frac{\rho_{s}}{\rho_{f}} \frac{\partial w^{2}}{\partial t^{2}}=\frac{L^{2} F_{f}}{D B R_{s d}}+\left(\frac{\rho_{s}}{\rho_{f}}-1\right) F r^{-1}$

where $l$ is the coordinate variable along the fiber length direction; $w$ is the displacement, due to bending, perpendicular to the fiber length and width directions; $F_{f}$ is the non-dimensional hydrodynamic force distributed on the fiber per unit length; $D$ is the thickness of the fiber; $B$ is its width; $g$ is the gravity acceleration; $\mathrm{Fr}=V_{0}^{2} /(\mathrm{Lg})$ is Froude number, which is the ratio of the fluid inertia to the gravity. It is seen that the first term of the left side of Eq. (31) is related to fiber rigidity and the second term is a fiber inertial term while the first term of the right side is associated with the hydrodynamic force and the second term comes from gravity or buoyancy force. The fiber deformation and motion can be determined by the rigidity $E I^{*}$, the fiber and fluid inertia, hydrodynamic force, and the gravity force. The gravity force drives fibers to settle down vertically and the fibers may be deformed due to their elasticity while the fiber rigidity and fluid forces may resist the deformation.

A non-dimensional rigidity-to-gravity number $\beta$ is defined by a ratio of the rigidity $E I^{*}$ to the buoyancy force as

$\beta=\frac{E I^{*}}{\left(\rho_{s} / \rho_{f}-1\right) / F r}$.

A larger rigidity-to-gravity number may result in a smaller deformation and vice verse. This number is useful for analysis of effects 
T/6

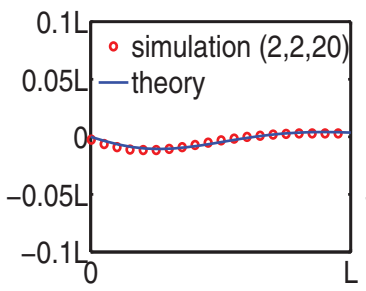

4T/6

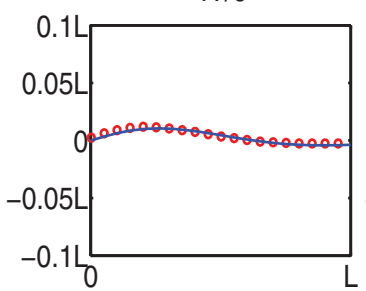

$\mathrm{T} / 6$

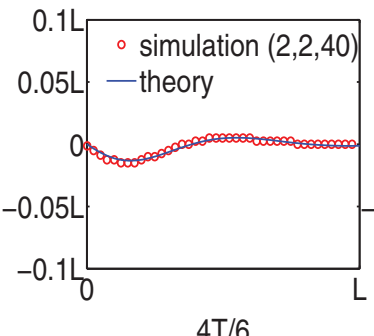

4T/6

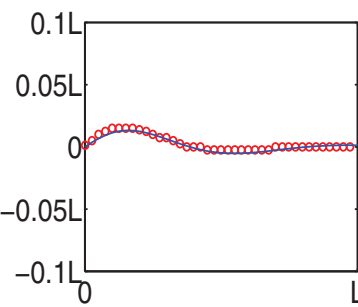

2T/6

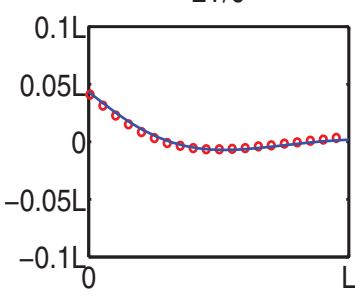

5T/6

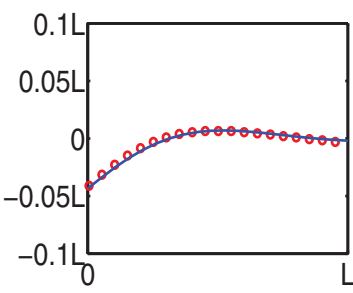

2T/6

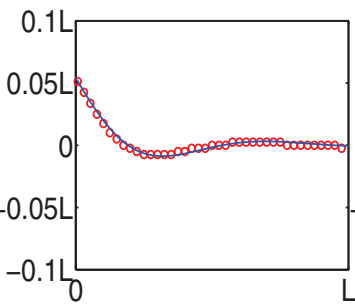

$5 T / 6$

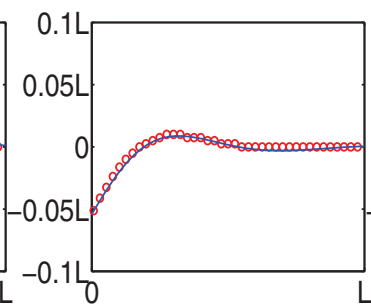

3T/6

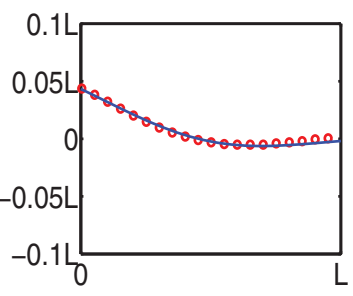

T

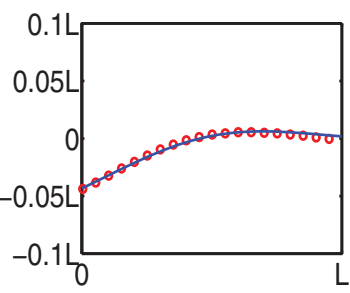

3T/6

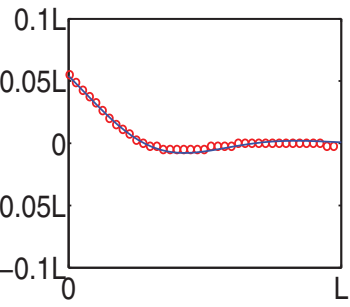

T

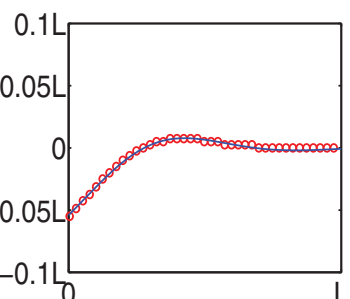

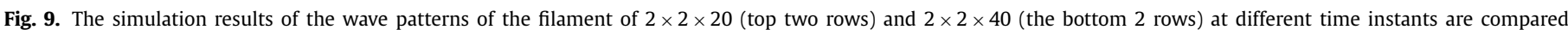

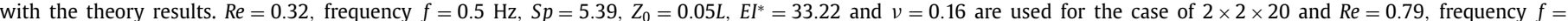
$0.25 \mathrm{~Hz}, S p=8.58, Z_{0}=0.0625 L, E I^{*}=5.32$ and $v=0.16$ are used for the case of $2 \times 2 \times 40$. The horizontal and vertical axis are normalized by the length of filament $L$.
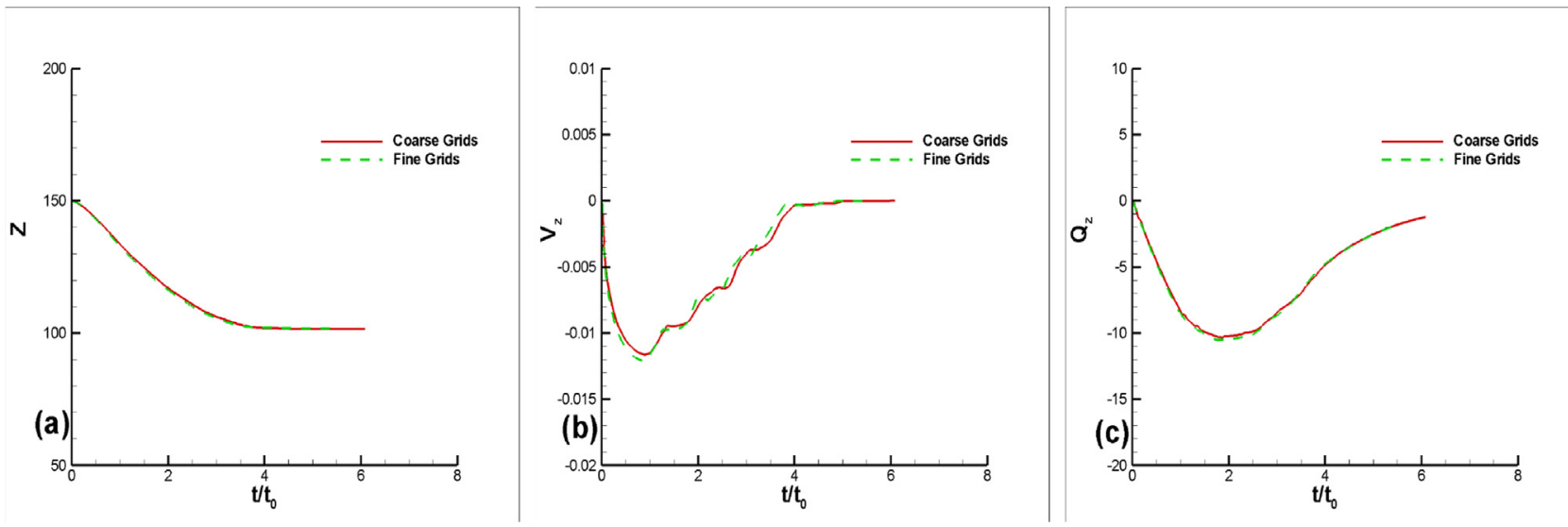

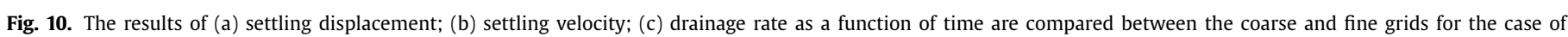

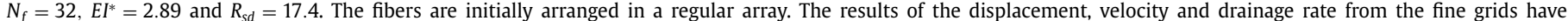
been scaled to be comparable with those from the coarse grids. 


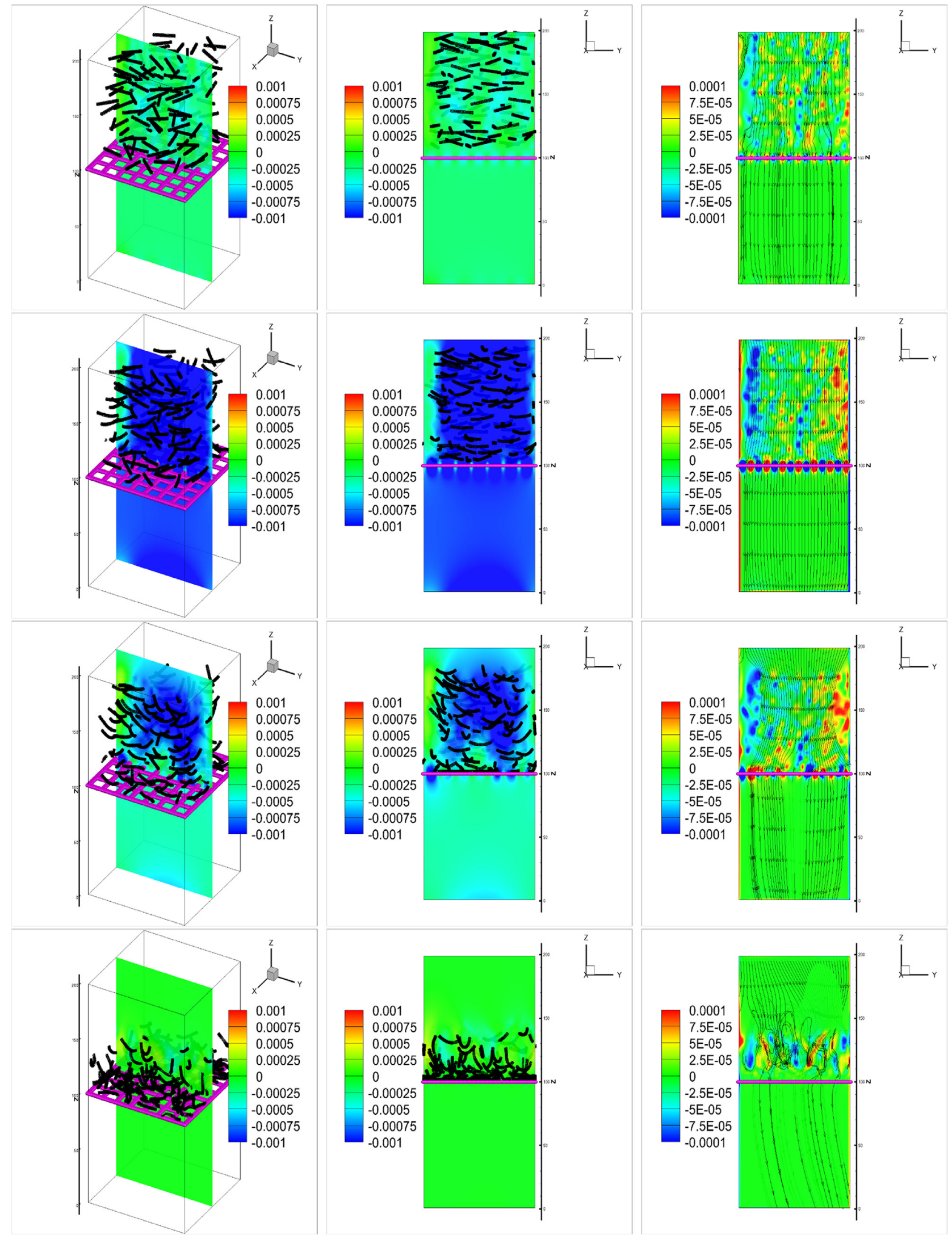

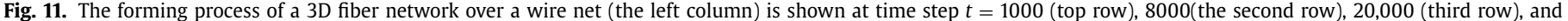

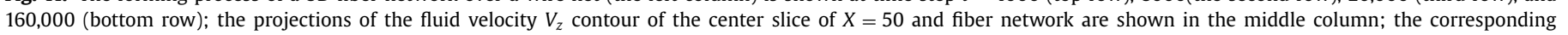

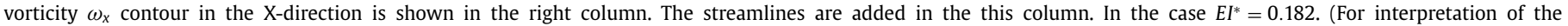
references to color in the text, the reader is referred to the web version of this article.) 
of the rigidity and gravity on the deformation. In the later stage of the sedimentation, a fiber mat has being built over the top surface of the fiber and the fiber mat structure may largely depend on fiber rigidity, concentration, fiber distribution, and specific surface area. More flexible fibers and a large fiber concentration may be packed with less porosity and lead to a large flow resistance and a smaller drainage rate. The water drainage rate will also be affected by screen structures, its opening ratio, ratio of screen-tofluid velocity, etc. This complex dynamic process cannot be easily expressed by an empirical formula, but can be evaluated by computer simulations using the present model.

\subsection{Simulation conditions}

To simulate drainage rate of a fiber network, a wire net with square openings is located at $Z=0.5 N_{z}$ as shown in Fig. 11 and can move in the horizontal or Y-direction with a relative screen/wire velocity $U_{w}$ to the average fluid velocity in the horizontal direction, which is equal to zero in the considered cases. If the screen speed is equal to the average fluid speed in the horizontal direction, or screen-to-fluid speed ratio is zero, the relative screen velocity $U_{w}=0$. However, if the screen velocity is larger or smaller than the average fluid velocity, the relative screen speed $U_{w}$ is nonzero. We can vary fiber concentrations $C$, value of $U_{w}$, fiber rigidity $E I^{*}$, and screen opening ratio $\Phi$ to study their influences on drainage rates. The current computing coordinate is selected to go with fluid flow. The gravity is set in the negative of the Z-direction. The drainage rate $Q_{z}$ can be calculated by the average fluid velocity $V_{z}$ in the gravity direction over all the wire openings multiplying the opening area. For the fluids domain, periodic boundary conditions are imposed in all directions. For solid fibers, the periodic conditions are also imposed except in the Z-direction or gravity direction.

In order to examine the resolution of grids, two levels of grids are exercised. In the coarse grid, a fiber size of $2 \times 2 \times 20$ and a simulation box of $100 \times 100 \times 200$ are used; in the fine grids, a fiber size of $3 \times 3 \times 30$ and a simulation box size of $150 \times 150 \times 300$ are employed. Thus, the unit length in the fine grids is 1.5 times smaller than that in the coarse grids and the time step length is 2.25 times smaller than the coarse grids. The results of the settling displacement and velocity as a function of time as well as the drainage rate are compared between the coarse and fine grids for the case of fiber number $N_{f}=32$ and $R_{s d}=17.4$ in Fig. 10, where the maximum of the settling velocity of the mass center of all fibers is used as a reference velocity, i.e. $V_{0}=V_{0 z}$ when the particle Reynolds number $R_{s d}$ is calculated. The difference between two results is not significant, in particular, for the drainage rates. Therefore, to lower the computational load, the fiber size of $2 \times 2 \times 20$ and the simulation box size of $100 \times 100 \times 200$ are selected. In the simulations, one lattice unit length is $2.5 \times 10^{-3} \mathrm{~cm}$; one time step interval is $1.25 \times 10^{-5} \mathrm{~s}$.

First, a random fiber network is built. Three random numbers $\mathrm{X}, \mathrm{Y}, \mathrm{Z}$ are utilized to have the random positions of the mass center of each fiber and additional two random numbers, $\phi$, defined as the angle between the projection of the fiber axis on the XYplane with the X-direction, and $\theta$, defined as the angle of the fiber axis with the XY-plane, are employed to create in-plane angle and Z-direction angle of fibers. $\phi$ ranges from $0^{\circ}$ through $360^{\circ}$ and $\theta$ ranges from $-15^{\circ}$ through $15^{\circ}$. All the initial positions of fibers are distributed above the screen. To ensure that there is no overlap between any of two fibers, the distance between each two solid particles is measured. If the distance is less than a value, say, 3 lattice units, the fiber should be reconstructed. Up to 500 fibers are used in the simulations.

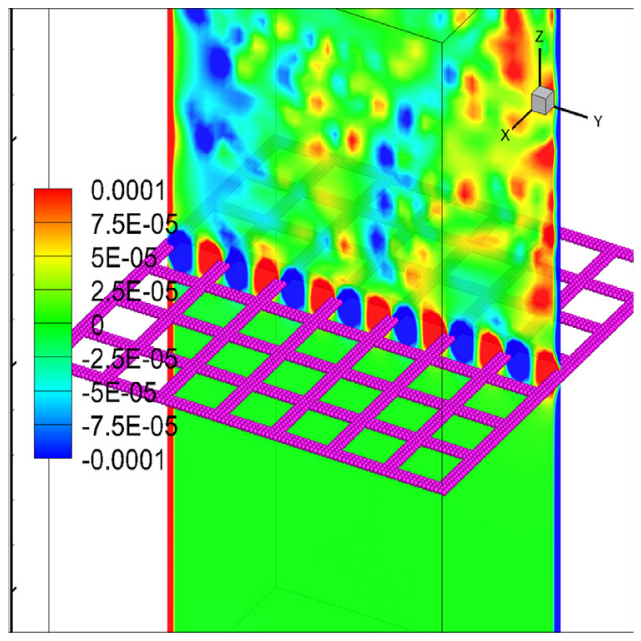

Fig. 12. The contours of the vorticity $\omega_{x}$ on the center slice at $X=50$ are shown for the same figure as the right column at $t=8000$ of Fig. 11 except in a magnified three dimensional space. (For interpretation of the references to color in the text, the reader is referred to the web version of this article.)

\subsection{Fluid fields and fiber network}

An example of fiber networks in the simulation coordinate is shown in Fig. 11 where the results of a forming process of a fiber network settling on the forming wire at four different time steps are illustrated for the case of 500 fibers with a rigidity of $E I^{*}=0.182$. In this case the fiber concentration in weight is about $2.4 \%$, the screen-to-fluid velocity ratio $U_{w}=0$, the wire opening ratio $\Phi=0.71$ and $R_{s d}=3.872$.

There are down flows with blue color at $t=1000$ although up flows with yellow colors are observed. The fluid flows down and goes through the wire screen. The blue color along the screen line near $Z=100$, as shown in the center column of Fig. 11, demonstrate the fluid flowing through the screen holes. After passing the screen opening, the down flows are reduced. At $t=8000$ (see the second row in Fig. 11), the down flows (blue color) dominate the up flows and the velocity of the flows through the screen holes increases, due to the gravitational force, is much larger than that in the earlier stage of $t=1000$ and becomes maximum. At $t=20,000$, the velocity of the down flow is reduced due to the resistant of the screen when the fibers approach the screen surface. At $t=160,000$, all the fibers migrate close to the top surface of the screen, a fiber mat has been built up over the screen, increases the resistant, and slows down the fluid flowing, the drainage rate is greatly reduced. The corresponding vorticity contours in the Xdirection are shown in the right column. In the initial stage at $t=1000$, a lot of small vortexes are formed over all the space. At $t=8000$, vortices increase and become larger as the settling velocity increases. There are lined vorticity along the screen surface, each vorticity has alternative directions [blue color (negative sign) after red color (positive sign)] due to two back flows in the wall area of a hole, where the geometrical shape is sharply changed, causes flow separation from the hole wall and results in a vorticity pair, each having opposite sign as shown in Fig. 12. There are seven vorticity pairs in total for the seven holes (see the right column of Fig. 11). The intensity and the size of these vorticity increase at $t=8000$ when the drainage rate arrives at a maximum. After passing the maximum point, the intensity of vorticity is reduced at $t=20,000$. After a fiber mat is formed at $t=160,000$, the vorticity is very small due to the settled fibers and their reduced velocity.

When the fiber falls down vertically and has a large settling velocity, it pushes the fluid in the front side, which generates a 


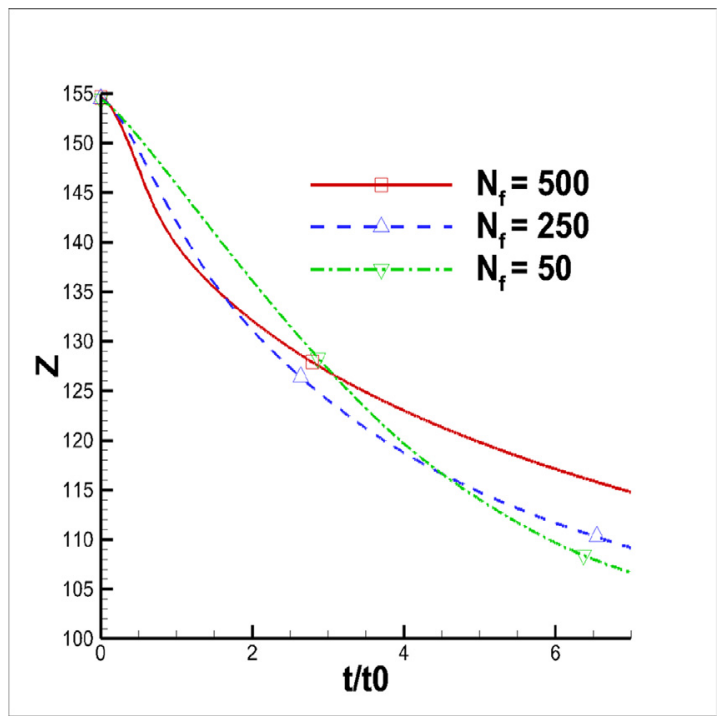

Fig. 13. Comparison of the settling position of the mass center of all fibers as a function of time among the cases of $N_{f}=50,250$, and 500. The wire net opening ratio $\Phi=0.58$. The time is normalized by $t_{0}=L / V_{0}$.

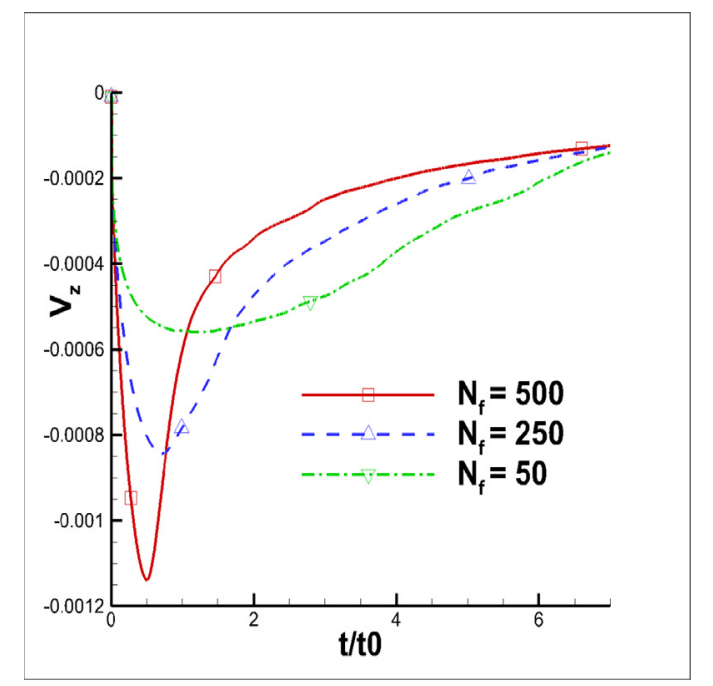

Fig. 14. Comparison of the settling velocity of the mass center of all fibers as a function of time among the cases of $N_{f}=50,250$ and 500. The wire net opening ratio $\Phi=0.58$.

higher pressure in the front side and a lower pressure in the back. The fluid tries to flow from the high pressure area (the front of the fiber) to the low pressure area (the back of the fiber). The fluid in the center part of the fiber does not easily pass the solid fiber and flow to the back area. However, the fluid in the areas of the two ends of fiber does easily flow to the back through the fiber tip areas and creates a back flow in a circular manner due to fluid separation, so called tip vortex. There are two vortices around the ends of the fiber and the vortices rotate in the opposite direction. This basic mechanism induces vortices. The right columns of Fig. 11 show that there are a large number of vortices at $t=8000$ when the fiber settling velocities are large and the vortices are almost disappeared at $t=160,000$ when the fibers approach the stationary screen and the settling velocities are largely reduced. Obviously, the vortices are associated with large settling velocities or momentum.

Other observation is that most fibers are obviously curved and have concave upward shapes during settling due to their flexibility

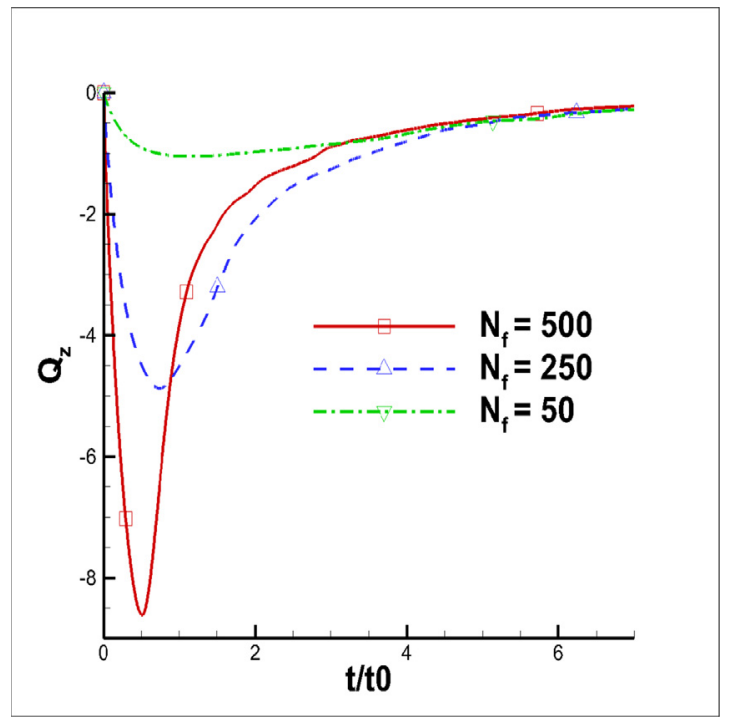

Fig. 15. Comparison of the drainage rate of the fiber network as a function of time among the cases of fiber number $N_{f}=50,250$ and 500 . The wire net opening ratio $\Phi=0.58$.

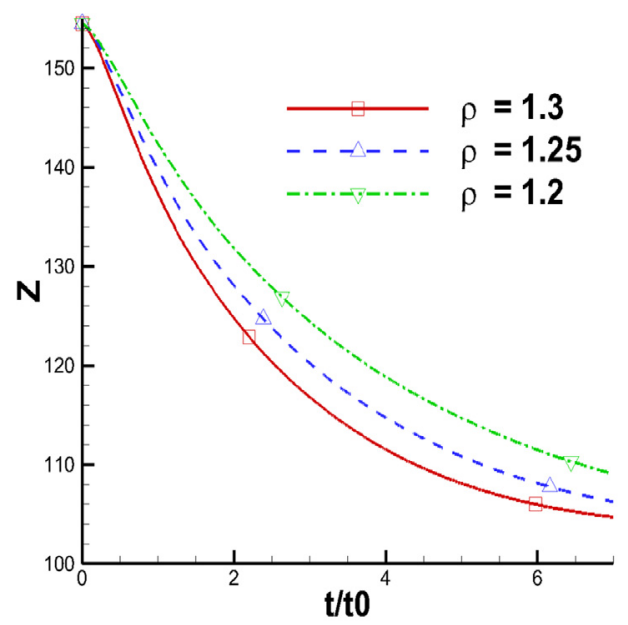

Fig. 16. Comparison of the position of the mass center of all fibers as a function of time among the different densities of fibers. In these cases, $E I^{*}=13.37, N_{f}=250$ and $\Phi=0.54$.

(see Fig. 11 the second and third rows at $t=8000$ and 20,000). For this case, the rigidity-to-gravity number $\beta=0.0018$ is much smaller than 1 , indicating that the gravity driving force dominates the rigidity and causes a large deformation.

In addition, the long bodies of most fibers are mainly oriented horizontally, similar to rigid fiber cases (Huang et al., 1994). The pressure torques of fluid always turn the broadside of the fibers in the horizontal direction. This is due to fluid inertial effects. The fiber in a Stokes fluid does not have this property.

\subsection{Effects of fiber concentrations}

To find how the fiber concentration affects the drainage rate, the number of fibers is varied at level of $N_{f}=50,250$ and 500 while keeping other simulation conditions same. These numbers of fibers correspond to fiber weight concentrations of $0.24 \%, 1.2 \%$ and $2.4 \%$ respectively. The simulation results are shown in Fig. 13 for the position in the Z-direction of the mass center of all fibers 


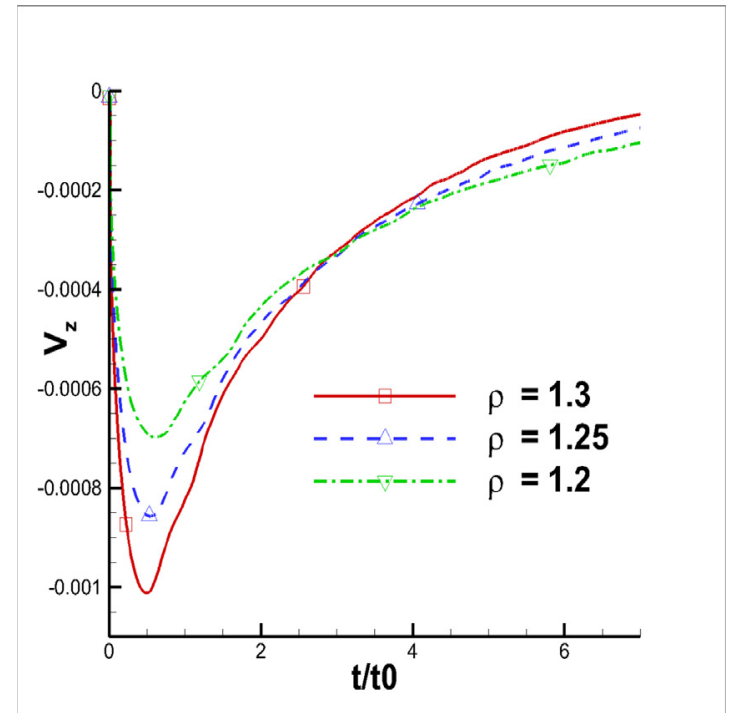

Fig. 17. Comparison of the settling velocity of the mass center of the fiber network as a function of time among the different densities of fibers. In these case, $E I^{*}=$ 13.37, $N_{f}=250$ and $\Phi=0.54$.

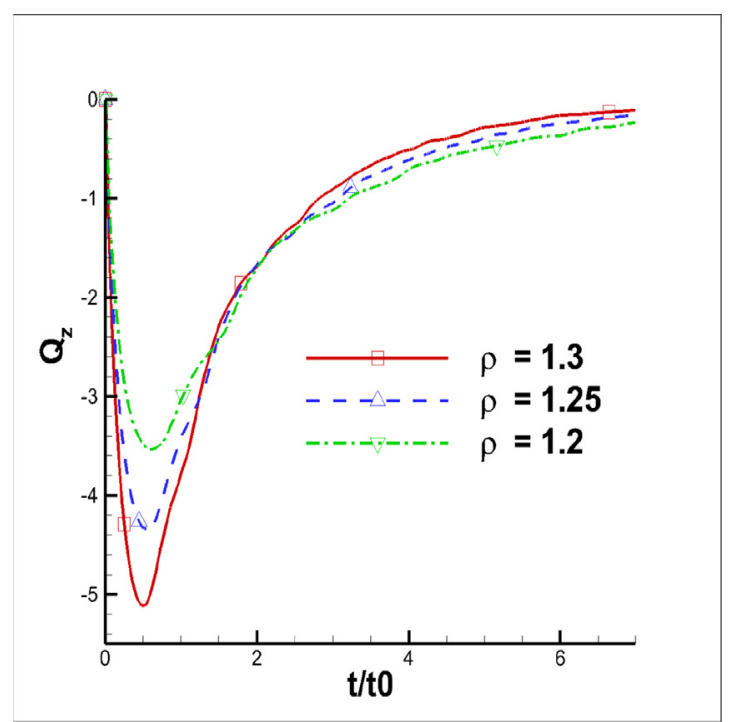

Fig. 18. Comparison of the drainage rate of the fiber network as a function of time among the different densities of fibers. In these case, $E I^{*}=13.37, N_{f}=250$ and $\Phi=$ 0.54 .

as a function of time, in Fig. 14 for the settling velocity of the mass center of the fiber network, and in Fig. 15 for the drainage rate. Fig. 13 shows that the curve of the Z-position of fiber network reduces monotonically with different slopes or settling velocities. Theses curves finally become flat at different levels in the Z-direction, demonstrating that a thicker fiber mat is formed for a larger number of fibers.

Fig. 14 shows that the velocity of the fiber network in the gravity or Z-direction increases non-linearly, arrives at a maximum around time step $t / t_{0}=0.5$ for the case of the fiber number $N_{f}=500$, then reduces to zero until the fiber mat setting on the screen. As the number of the fibers increases, the settling velocity increases faster and the value of the maximum increases. Also, the peak of the maximum delays as the number of fiber reduces. It seems that the higher the fiber concentration, the larger the settling velocity and the faster the fiber mat forms. The nonlinear curves with a peak in the settling velocity reflect the fibers

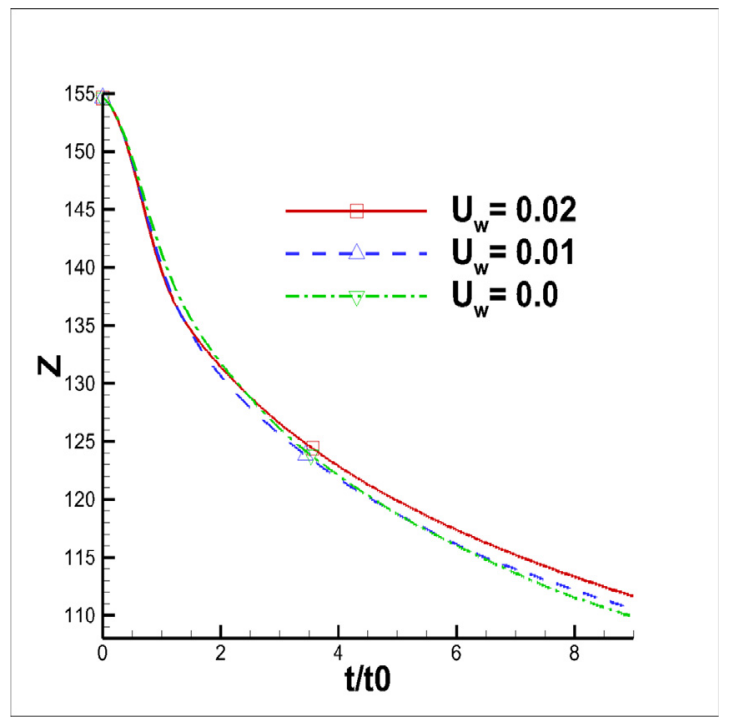

Fig. 19. Comparison of the settling position of the mass center of fiber network as a function of time among the different screen-to-fluid velocity ratios at the same screen opening ratio $\Phi=0.71$ and $E I^{*}=0.18$.

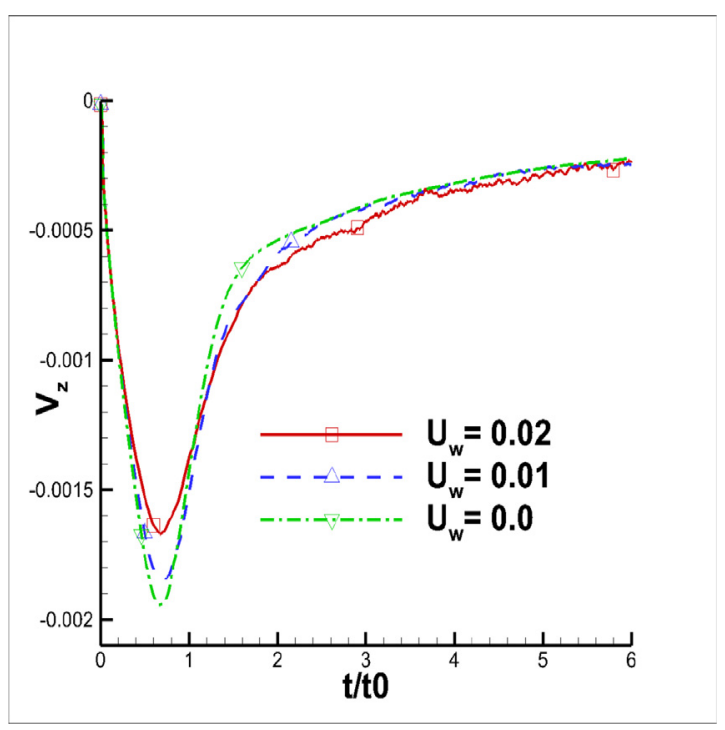

Fig. 20. Comparison of the settling velocity of the mass center of fiber network as a function of time among the different screen-to-fluid velocity ratio at the same screen as in Fig. 19. The right is a magnified figure of the circle area.

inertial effect. Since a larger number of fibers have a larger mass in total, they may settle faster due to its inertial effect. Similarly, Fig. 15 shows that the drainage rate is a nonlinear curve with respect to time. The drainage rate increases to a maximum, then reduces. The maximum is significantly larger for the case of $N_{f}=500$ than other cases, suggesting that the inertia of fiber network has a major contribution to the drainage rates of the fiber network. Again, the peak of the curve of $Q_{z}$ is delayed as the number of fibers reduces.

\subsection{Effects of fiber density}

To further verify the fiber inertial effect and provide a firm evidence, the ratios of the fiber to fluid density are varied at three different levels of $\rho=\frac{\rho_{s}}{\rho_{f}}=1.2,1.25$, and 1.3. while other conditions are kept the same. The results of the mass center of all the fibers, settling velocity, and drainage rate as a function of time are plot- 


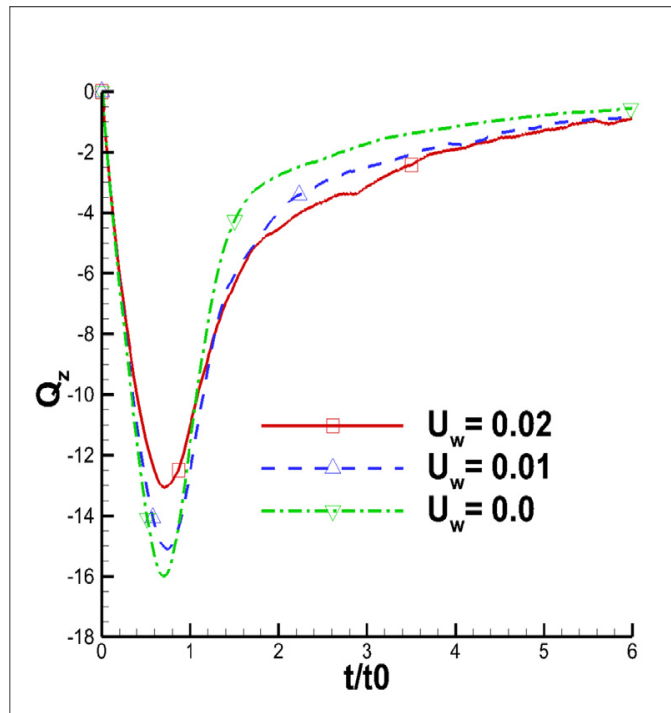

Fig. 21. Comparison of the drainage rate of the fiber network as a function of time among the different screen-to-fluid velocity ratios at the same screen as in Fig. 19.

ted in Figs. 16, 17 and 18, respectively. These figures clearly show that the settling velocity significantly increases as the fiber density increases. Thus, due to non-slip boundary condition, the boundary layer fluids in the immediate vicinity of the fiber surface will move along with the fibers and arrive at the top of the screen faster, resulting in a larger drainage rate. It is clear that the drainage rate increases as the fiber inertia increases.

\subsection{Effects of relative screen-to-fluid velocity ratio}

To study the effect of relative screen-to-fluid velocity ratio, $U_{w}=0.0,0.01$, and 0.02 are, respectively, imposed on the screen in the Y-direction, and computer simulations are conducted in the same conditions at the number of fiber $N_{f}=500$; the screen opening ratio $\Phi=0.71$, fiber density $\rho=1.2$, and the fiber rigidity of $E I^{*}=1.81$. Fig. 20 shows that the settling velocity is the largest for $U_{w}=0.0$ and the smallest for $U_{w}=0.02$ although the difference is not as large as the one in the case of the different number of fibers. The drainage rate is also the largest for $U_{w}=0.0$ as shown in Fig. 21. It seems that the smaller screen-to-fluid velocity ratio has a better drainage rate although the thickness of the fiber network is not too different (see Fig. 19). In other words, a relative speed of screen-to-fluid is not favorable for drainage rate. A larger shear force, caused by moving screen in the horizontal direction, around the area of the screen may alter the direction of the fluid flowing down and reduces the drainage rate. The results coincide with paper mill manufacture experience (Niskanen, 2008).

\subsection{Effects of fiber flexibility}

The simulations are carried out in the same conditions with $\rho=1.3$ except that the rigidity $E I^{*}$ is varied at four different levels. The results of settling position, velocity, and drainage rates of the fiber network as a function of time are plotted in Figs. 22, 23 and 24, respectively. Fig. 23 shows that the fibers with the lowest rigidity settle fastest but has the lowest drainage rate as shown in Fig. 24. The differences are small but distinguishable. We demonstrate that more flexible fibers fall down faster. Since the shape of the fiber body is an important factor for drag force, a more streamlined fiber body shape can reduce drag. When the fiber shape is relaxed due to its flexibility, the flow pattern is changed. When more fluids are directed along the streamlines, the pressure difference between the front and back area of the fiber is reduced, in particular, in the tip region and a drag reduction is induced. Thus, the fibers falling faster may bring more surface boundary water to the screen area and be favorable for drainage. On other hand, more flexible fibers may form a more compacted or flat fiber mat with smaller number of pores and smaller pore size, over the screen, due to conformability and flexibility. The compacted fiber network resists the fluid flowing and reduces the drainage rate. The large resistant effect of the compacted fiber network dominates the surface boundary water falling with the fiber. Therefore, the flexibility has a negative effect on drainage rate. The more compact fiber mat is reflected in Fig. 22, where the mass center of the fiber network with the lowest rigidity has the smallest $\mathrm{Z}$ value in the final stage, indicating the thickness of the fiber mat is smallest.

The rigidity-to-gravity numbers are calculated and the results are $\beta=0.37,0.074,0.015$, and 0.0029 for the four different levels of the rigidity $E I^{*}=36.76,7.38,1.46$ and 0.29 . It is reasonable that the smallest rigidity-to-gravity number case has the largest deformation during sedimenting process, the most compacted fiber mat with the smallest pores at the final stage, and the worst drainage rate.

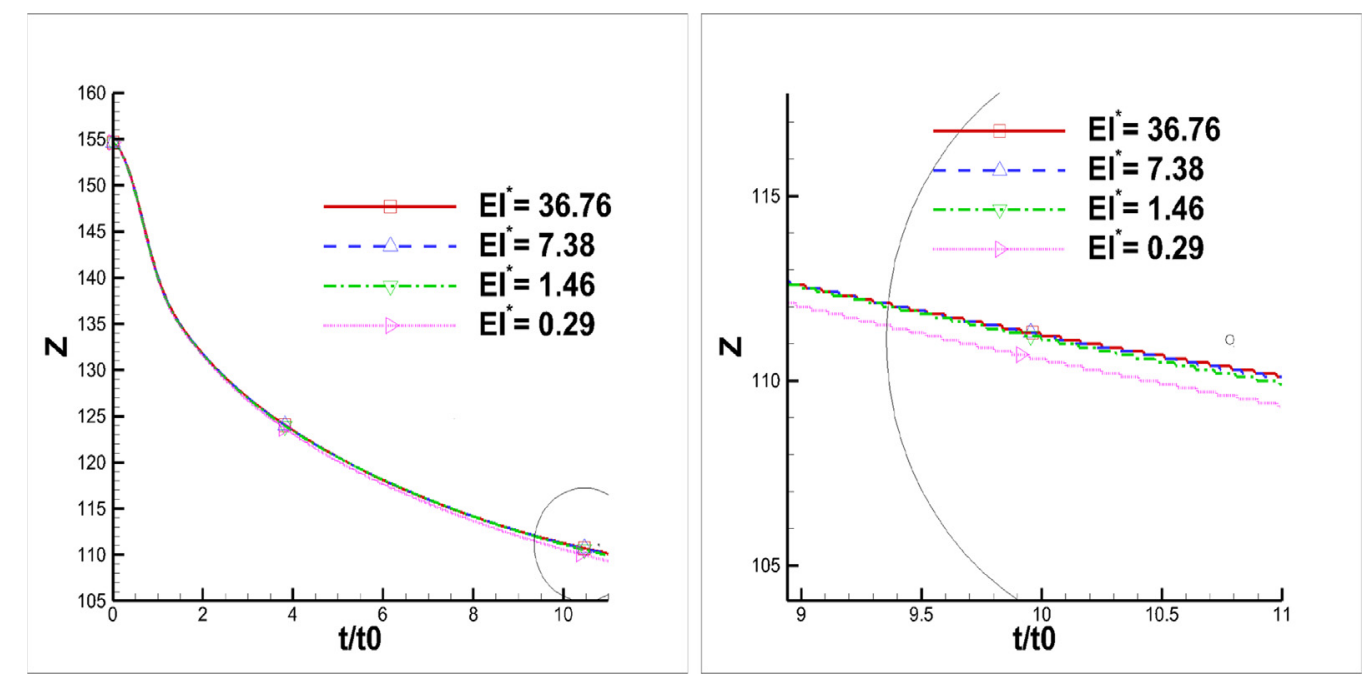

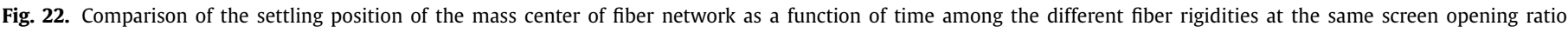
$\Phi=0.71$ and $N_{f}=500$. 


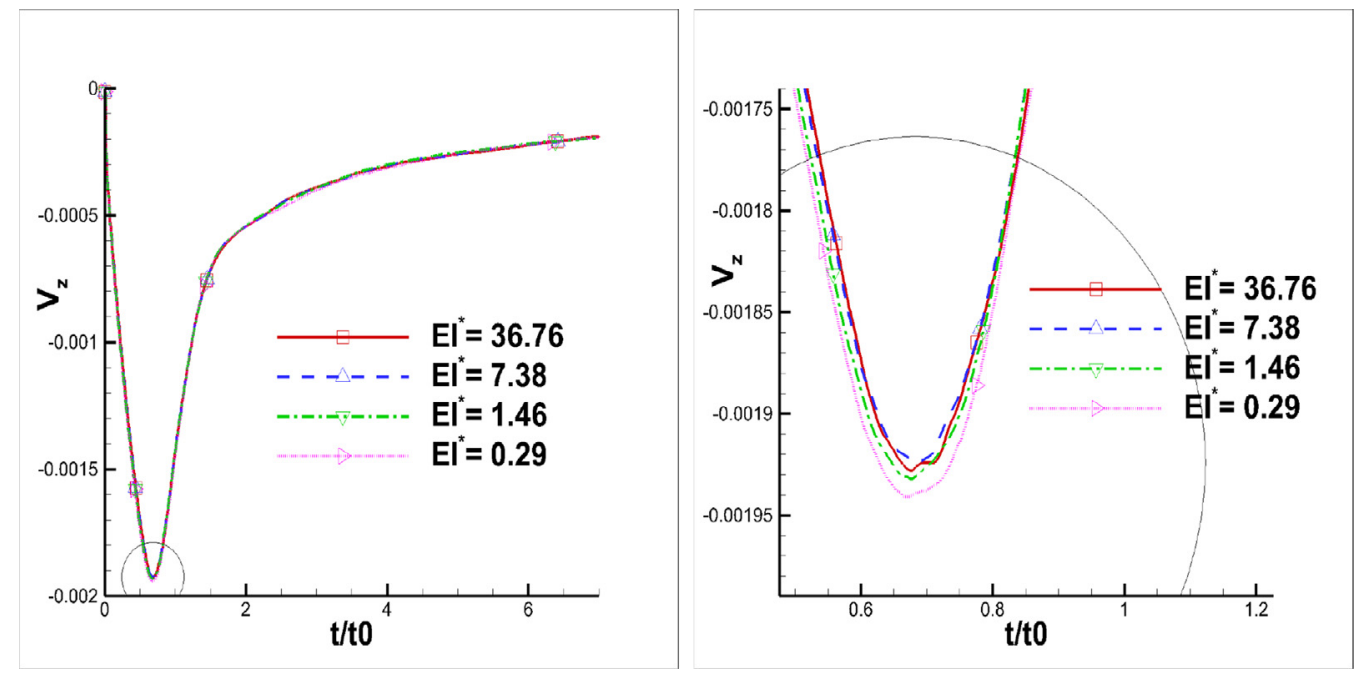

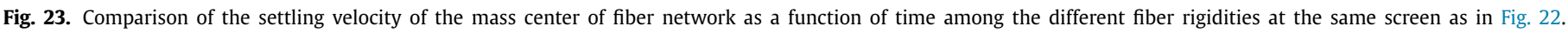
The right is a magnified figure of the circle area.

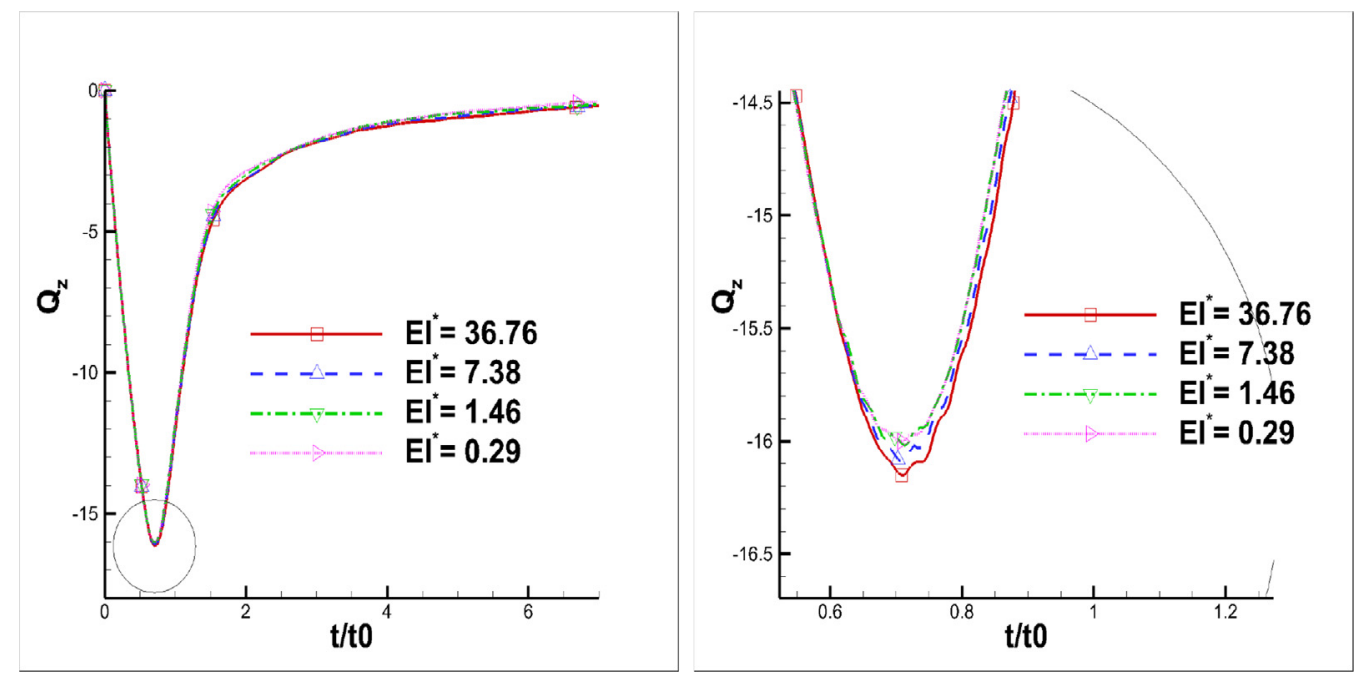

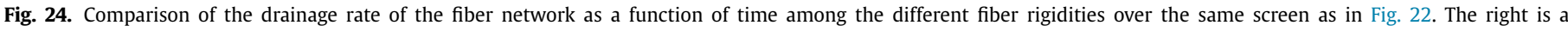
magnified figure of the circle area.

The findings concur with the current knowledge (Niskanen, 2008). We make a remark here that in all the simulations, the fiber surface areas are kept in the same and the flexibility is altered by varying the fiber bending rigidity only. It is pointed out that for a given mass, smaller fibers may have larger surface areas, receive larger drag force, and settle slower, as compared with larger fibers. Such the surface area effect is not included in the present work.

\section{Conclusion}

We demonstrated that a suspension with large numbers of flexible fibers can be accurately simulated by using lattice-Boltzmann and lattice-spring method. The direct forcing-force can be simply calculated through the Newtonian second law and imposed through immersed boundary scheme. The present method is validated and applied to simulate a large numbers of fibers setting on a moving screen/wire. It is revealed that drainage rate is a nonlinear function of time. The drainage rate rapidly increases to a maximum, then reduces to a low level after fiber mat is formed. As number of fibers increases, the drainage rate increases due to faster settling velocity of fibers, which may bring surface fluid flowing down. It is found that a large screen-to-fluid velocity ratio may have a negative effect on the drainage rate. The flexibility may also have a negative effect on drainage rate since more flexible fibers may form a more compact or flat fiber mat with less pores which resists fluid flowing, although they may settle faster.

\section{Acknowledgement}

Dr. Qi, Mr. Tang and Wu acknowledge the support from the National Science Foundation of USA under Award Number 1126438. Dr. He would like to thank the financial supports from the National Natural Science Foundation of China (NNSFC) under Award No. 11232011 (Key project) and the National Basic Research Program of China (973 Program) under Award No. 2013CB834100.

\section{References}

Aidun, C.K., Clausen, J.R., 2010. Lattice-Boltzmann method for complex flows. Annu. Rev. Fluid Mech. 42, 439-472.

Aidun, C.K., Lu, Y., Ding, E., 1998. Direct analysis of particulate suspensions with inertia using the discrete Boltzmann equation. J. Fluid Mech. 373, 287.

Allen, M.P., Tildesley, D.J., 1987. Computer Simulation of Liquids. Oxford Science Publication.

Basagaoglu, H., Allwein, S., Succi, S., Dixon, H., Carrola, J.T., 2013. Two and three-dimensional lattice-Boltzmann simulations of particle migration in microchannels. Microfluid. Nanofluid. 15, 785. 
Basagaoglu, H., Meakin, P., Succi, S., Redden, G.R., Ginn, T.R., 2008. Two-dimensional lattice Boltzmann simulation of colloid migration in rough-walled narrow flow. Phys. Rev. E 77, 031405.

Benzi, R., Succi, S., Vergassola, M., 1992. The lattice Boltzmann equation: theory and applications. Phys. Rep. 222, 145-179.

Bhatnagar, P.L., Groos, E.P., Krook, M., 1954. A model for collision processes in cases. I. Small amplitude processes in charged and neutral one-component systems. Phys. Rev. 94, 511-525.

Burgers, J., 1938. Second Report on Viscosity and Plasticity. North Holland Publishing Co.

Butler, J.E., Shaqfeh, E.S.G., 2002. Dynamic simulations of the inhomogenous sedimentation of rigid fibers. J. Fluid Mech. 468, 205-237.

Buxton, G.A., Verberg, R., Jasnow, D., Balazs, A.C., 2005. Newtonian fluid meets an elastic solid: coupling lattice Boltzmann and lattice-spring models. Phys. Rev. E 71, 056707.

Dahlkild, A.A., 2011. Finite wavelength selection for the linear instability of a suspension of settling spheroids. J. Fluid Mech. 689, 183-202.

Delouei, A.A., Nazari, M., Kayhani, M.H., Succi., S., 2014. Non-Newtonian unconfined flow and heat transfer over a heated cylinder using the direct-forcing immersed boundary thermal lattice Boltzmann method.. Phys. Rev. E 89, 053312.

Duenweg, B., Ladd, A.J.C., 2009. Lattice Boltzmann simulations of soft matter systems. Adv. Polym. Sci. 221, 89-166.

Feng, Z.G., Michaelides, E.E., 2004. The immersed boundary-lattice Boltzmann method for solving fluid-particle interaction problems. J. Comput. Phys. 195, 602-628.

Feng, Z.G., Michaelides, E.E., 2005. Poteus: a direct forcing method in the simulations of particulate flows. J. Comput. Phys. 202, 20-51.

Fogelson, A.L., Peskin, C.S., 1988. A fast numerical method for solving the three dimensional stokes equations in the presence of suspended particles. J. Comput. Phys. 79, 50-69.

Forgacs, O., Mason, S., 1959. Particle motions in sheared suspensions: IX. Spin and deformation of threadlike particles. J. Colloid Sci. 14 (5), 457-472.

Forgacs, O., Mason, S., 1959. Particle motions in sheared suspensions: X. Orbits of flexible threadlike particles. J. Colloid Sci. 14 (5), 473-491.

Gao, T., Hu, H.H., Castaneda, P.P., 2011. Rheology of a suspension of elastic particles in a viscous shear flow. J. Fluid Mech. 687, 209-237.

Gao, T., Hu, H.H., Castaneda, P.P., 2013. Dynamics and rheology of elastic particles in an extensional flow. J. Fluid Mech. 715, 573-596.

Glowinski, R., Pan, T.W., Hesla, T.I., Joseph, D.D., 1999. A distributed Lagrange multiplier/fictitious domain method for particulate flows. Int. J. Multiphase Flow 25, 755-795.

Glowinski, R., Pan, T.W., Hesla, T.I., Joseph, D.D., Periaux, J., 2001. A fictitious domain approach to the direct numerical simulation of incompressible viscous flow past moving rigid bodies: application to particulate flow. J. Comput. Phys. 169, 363-426.

Guo, Z., Zheng, C., Shi, B., 2002. Discrete lattice effects on the forcing term in the lattice Boltzmann method. Phys. Rev. E 65, 046308.

He, X., Shan, X., Doolen, G.D., 1998. Discrete Boltzmann equation model for nonideal gases. Phys. Rev. E 57, R13.

Hu, H.H., 1996. Direct simulations of flows of solid-liquid mixtures. J. Multiphase Flow 22, 335-352.

Huang, P.Y., Feng, J., Joseph, D.D., 1994. The turning couples on an elliptic particle settling in a vertical channel. J. Fluid Mech. 271, 1-16.

Joung, C., Phan-Thien, N., Fan, X., 2001. Direct simulation of flexible fibers. J. Non-Newton. Fluid Mech. 99 (1), 1-36

Kang, S.K., Hassan, Y.A., 2011. A comparative study of direct-forcing immersed boundary-lattice Boltzmann methods for stationary complex boundaries. Int. J. Numer. Methods Fluids 66, 1132-1158.

Koch, D.L., 1994. Hydrodynamic diffusion in a suspension of sedimenting point particles with periodic boundary conditions. Phys. Fluids 6, 2894.

Koch, D.L., Ladd, A.J., 1997. Moderate Reynolds number flows through periodic and random arrays of aligned cylinders. J. Fluid Mech. 349, 31.

Koch, D.L., Shaqfeh, E.S.G., 1989. The instability of a dispersion of sedimenting spheroids. J. Fluid Mech. 209, 521-542.

Koch, D.L., Shaqfeh, E.S.G., 1991. Screening in sedimenting suspensions. J. Fluid Mech. 224, 275-303.

Kuusela, E., Hofler, K., Schwarzer, S., 2001. Computation of particle settling speed and orientation distribution in suspensions of prolate spheroids. J. Eng. Math. 41, 221-235.

Ladd, A.J., 1994. Numerical simulations of particulate suspensions via a discretized Boltzmann equation. Part 1 theoretical foundation. J. Fluid Mech. 271, 285.

Lindström, S.B., Uesaka, T., 2007. Simulation of the motion of flexible fibers in viscous fluid flow. Phys. Fluids 19 (11), 113307.
Lindström, S.B., Uesaka, T., 2008. Simulation of semidilute suspensions of non-Brownian fibers in shear flow. J. Chem. Phys. 128 (2), 024901.

Luo, Y., hsien Wu, T., Oi, D., 2017. Lattice-Boltzmann lattice-spring simulations of flexibility and inertial effects on deformation and cruising reversal of self-propelled flexible swimming bodies. Comput. Fluids 155, 89-102.

Niskanen, K., 2008. Paper Physics. TAPPI.

Patankar, N.A., Singph, P., Joseph, D.D., Glowinski, R., Panc, T.W., 2000. A new formulation of the distributed Lagrange multiplier/fictitious domain method for particulate fows. Int. J. Multiphase Flow 26, 1509-1524.

Peskin, C.S., 1977. Numerical analysis of blood flow in the heart. J. Comput. Phys. 25 220-252.

Peskin, C.S., 2002. The immersed boundary method. Acta Numerica 11, 479-517.

Qi, D., 1999. Lattice Boltzmann simulations of particles in non-zero Reynolds number flows. J. Fluid Mech. 385, 41.

Qi, D., 2006. Direct simulations of flexible cylindrical fiber suspensions in finite Reynolds number flows. J. Chem. Phys. 125, 114901-114910.

Qi, D., Gordnier, R., 2015. Effects of deformation on lift and power efficiency in a hovering motion of a chord-wise flexible wing. J. Fluids Struct. 54, 142-170.

Qi, D., He, G., Liu, Y., 2014a. Lattice Boltzmann simulations of a pitch-up and pitchdown maneuver of a chord-wise flexible wing in a free stream flow. Phys. Fluids 26, 021902.

Qi, D., He, G., Liu, Y., 2014b. Lattice Boltzmann simulations of sedimentation of a single fiber in a weak vertical shear flow. Phys. Fluids 25, 093302.

Qi, D., Liu, Y., Shyy, W., Aono, H., 2010. Simulations of dynamics of plunge and pitch of a three-dimensional flexible wing in a low Reynolds number flow. Phys. Fluids 22, 091901.

Saintillan, D., Shaqfeh, E.S.G., Darve, E., 2006. The growth of concentration fluctuations in diluted dispersions of orientable and deformable particles under sedimentation. J. Fluid Mech. 553, 347-388.

Salahuddin, A., Wu, J., Aidun, C., 2012. Numerical study of rotational diffusion in sheared semidilute fibre suspension. J. Fluid Mech. 692, 153.

Salahuddin, A., Wu, J., Aidun, C., 2013. Study of semidilute fibre suspension rheology with lattice-Boltzmann method. Rheol. Acta 52 (10-12), 891-902.

Shin, M., Koch, D.L., Subramanian, G., 2006. A pseudospectral method to evaluate the fluid velocity produced by an array of translating slender fibers. Phys. Fluids 18, 063301

Shin, M., Koch, D.L., Subramanian, G., 2009. Structure and dynamics of dilute suspensions of finite-Reynolds-number settling fibers. Phys. Fluids 21, 123304.

Stockie, J.M., Green, S.I., 1998. Simulating the motion of flexible pulp fibres using the immersed boundary method. J. Comput. Phys. 147 (1), 147-165.

Succi, S., 2001. The Lattice Boltzmann Equation: For Fluid Dynamics and Beyond. Oxford Univ. Press, New York.

Succi, S., 2015. Lattice Boltzmann 2038. EPL 109, 50001.

Wan., D., Turek., S., 2006. Direct numerical simulation of particulate flow via multigrid fem techniques and the fictitious boundary method. Int. J. Numer. Methods Fluids 51, 531-566.

Whittington, S.G., 1998. Numerical Methods for Polymeric Systems, IMA Volumes in Mathematics and Its Applications. Springer, Berlin.

Wiggins, C.H., Riveline, D., Ott, A., Goldstein, R.E., 1998. Trapping and wiggling: elastohydrodynamics of driven microfilaments. Biophys. J. 74, 1043-1060.

Wu, J., Aidun, C., 2010a. A numerical study of the effect of fibre stiffness on the rheology of sheared flexible fibre suspensions. J. Fluid Mech. 662, 123.

Wu, J., Aidun, C.K., 2010b. A method for direct simulation of flexible fiber suspensions using lattice Boltzmann equation with external boundary force. Int. J. Multiphase Flow 36 (3), 202-209.

Wu, J., Aidun, C.K., 2010c. Simulating 3d deformable particle suspensions using lattice Boltzmann method with discrete external boundary force. Int. J. Numer. Methods Fluids 62, 765-783.

Wu, J., Shu, C., 2010. Particulate flow simulation via a boundary condition-enforced immersed boundary-lattice Boltzmann scheme. Commun. Comput. Phys. 7, 793-812.

Wu, T.H., Qi, D., 2017. Lattice-Boltzmann lattice-spring simulations of influence of deformable blockages on blood fluids in an elastic vessel computers and fluids. Comput. Fluids 155, 103-111.

Wu, T.H., Guo, R.S., He, G.W., Liu, Y.M., Qi, D., 2014. Simulation of swimming of a flexible filament using the generalized lattice-spring lattice Boltzmann method. J. Theor. Biol. 349, 1-11.

Wu, T.-H., Khani, M., Sawalha, L., Springstead, J., Kapenga, J., Qi, D., 2017. A cuda-based implementation of a fluid-solid interaction solver: the immersed boundary lattice-Boltzmann lattice-spring method. Commun. Comput. Phys. In press.

Zhang, F., Dahlkild, A.A., Lundell, F., 2013. Nonlinear disturbance growth during sedimentation in dilute fibre suspensions. J. Fluid Mech. 719, 268-294. 